OPEN ACCESS

Edited by:

Paul Sellin,

University of Surrey, United Kingdom

Reviewed by: Enrico Junior Schioppa, University of Salento, Italy David Pennicard, Helmholtz Association of German Research Centers (HZ), Germany

*Correspondence:

Lucio Anderlini

Lucio.Anderlini@fi.infn.it

Specialty section:

This article was submitted to Radiation Detectors and Imaging,

a section of the journal

Frontiers in Physics

Received: 31 July 2020 Accepted: 22 September 2020 Published: 04 November 2020

Citation:

Anderlini L, Bellini M, Bizzeti A, Cardini A, Ciaranfi R, Corsi C, Garau M, Lai A, Lagomarsino S, Lampis A, Loi A, Lucarelli C, Mariani S, Minafra N, Morozzi A, Mulargia R, Passaleva G, Passeri D, Sciortino S, Vecchi S and

Veltri M (2020) Fabrication and Characterisation of 3D Diamond Pixel

Detectors With Timing Capabilities.

Front. Phys. 8:589844.

doi: 10.3389/fphy.2020.589844

\section{Fabrication and Characterisation of 3D Diamond Pixel Detectors With Timing Capabilities}

Lucio Anderlini ${ }^{1 *}$, Marco Bellini ${ }^{2}$, Andrea Bizzeti ${ }^{1,3}$, Alessandro Cardini ${ }^{4}$, Roberto Ciaranfi ${ }^{1}$, Chiara Corsi ${ }^{5}$, Michela Garau ${ }^{4,6}$, Adriano Lai ${ }^{4}$, Stefano Lagomarsino ${ }^{1}$, Andrea Lampis ${ }^{4,6}$, Angelo Loi $^{4}$, Chiara Lucarelli ${ }^{1,7}$, Saverio Mariani ${ }^{1,7}$, Nicola Minafra ${ }^{8}$, Arianna Morozzi ${ }^{9}$, Roberto Mulargia ${ }^{10,11}$, Giovanni Passaleva ${ }^{1}$, Daniele Passeri ${ }^{9,12}$, Silvio Sciortino ${ }^{1,2,7}$, Stefania Vecchi ${ }^{13}$ and Michele Veltri ${ }^{1,14}$

${ }^{1}$ Istituto Nazionale di Fisica Nucleare-Sezione di Firenze, Firenze, Italy, ${ }^{2}$ Istituto Nazionale di Ottica, Consiglio Nazionale delle Ricerche, Firenze, Italy, ${ }^{3}$ Dipartimento di Scienze Fisiche, Informatiche e Matematiche, Università degli Studi di Modena e Reggio Emilia, Modena, Italy, ${ }^{4}$ Istituto Nazionale di Fisica Nucleare-Sezione di Cagliari, Cagliari, Italy, ${ }^{5}$ Laboratorio Europeo Spettroscopie Non Lineari, Sesto Fiorentino, Italy, ${ }^{6}$ Dipartimento di Fisica, Università degli Studi di Cagliari, Cagliari, Italy, ${ }^{7}$ Dipartimento di Fisica e Astronomia, Università degli Studi di Firenze, Firenze, Italy, ${ }^{8}$ Department of Physics and Astronomy, College of Liberal Arts and Science, The University of Kansas, Lawrence, KS, United States, ${ }^{9}$ Istituto Nazionale di Fisica Nucleare-Sezione di Perugia, Perugia, Italy, ${ }^{10}$ Istituto Nazionale di Fisica Nucleare-Sezione di Genova, Genova, Italy, ${ }^{11}$ Dipartimento di Fisica, Università degli Studi di Genova, Genova, Italy, ${ }^{12}$ Dipartimento di Ingegneria, Università degli Studi di Perugia, Perugia, Italy, ${ }^{13}$ Istituto Nazionale di Fisica Nucleare - Sezione di Ferrara, Ferrara, Italy, ${ }^{14}$ Dipartimento di Scienze Pure e Applicate, Università degli Studi di Urbino, Urbino, Italy

Diamond sensors provide a promising radiation hard solution to the challenges posed by the future experiments at hadron machines. A 3D geometry with thin columnar resistive electrodes orthogonal to the diamond surface, obtained by laser nanofabrication, is expected to provide significantly better time resolution with respect to the extensively studied planar diamond sensors. We report on the development, production, and characterisation of innovative 3D diamond sensors achieving 30\% improvement in both space and time resolution with respect to sensors from the previous generation. This is the first complete characterisation of the time resolution of 3D diamond sensors and combines results from tests with laser, $\beta$ rays and high energy particle beams. Plans and strategies for further improvement in the fabrication technology and readout systems are also discussed.

Keywords: tracking, diamond, 3D, pixel, sensors, nanofabrication

\section{INTRODUCTION}

The development of the particle detectors for future hadronic machines, such as the HighLuminosity Large Hadron Collider (HL-LHC) at CERN, faces the major challenge of reconstructing the trajectories (tracks) of an unprecedented density of charged particles. Stateof-the-art spatial granularity in the measurement of the energy deposits (hits) will be barely sufficient to distinguish tracks, and good time resolution will be necessary to distinguish the many hadronic collisions, or primary vertices, originated in a single bunch crossing [1,2]. Time information has been demonstrated to be very beneficial in pattern recognition procedures especially in events with a large number of tracks, allowing for complete $4 \mathrm{D}$ tracking algorithms, possibly implemented on programmable gate array devices [3].

The time resolution of a detector is defined by three main contributions: variations of the shape of the signal due to inhomogeneities in the charge generation and collection mechanisms; a time walk 
contribution related to the signal processing technique; and a jitter contribution due to the electronic noise superposed to the signal (see, e.g., Ref. [4] for a more detailed discussion).

The development of silicon pixel detectors aiming at excellent resolution on timing measurements has been extremely active in recent years, mainly focusing on Low-Gain Avalanche Detectors (LGADs) $[5,6]$. Best time resolutions are obtained with extremely thin LGAD sensors exploiting multiplication in a heavily doped $1-2 \mu \mathrm{m}$ thick layer. Indeed, by reducing the thickness of the LGAD detectors, the contribution from Landau fluctuations can be reduced preserving good signal-to-noise ratio by means of charge multiplication. For example, time resolutions of the order of $30 \mathrm{ps}$ per layer have been reported using $50 \mu \mathrm{m}$ LGAD detectors [7].

The higher density of ionising particles expected at future experiments also represents a serious concern for the radiation damage of both sensors and front-end electronics. For example, the sensors in the innermost part of the vertex detector of the upgraded LHCb experiment planned for taking data from 2032 on, will have to cope with a radiation dose of the order of $10^{17}$ $1 \mathrm{MeV} n_{e q} \mathrm{~cm}^{-2}$ for an exposure to radiation estimated to last ten years [8]. Potential flavour physics experiments at future hadron collider will also face the same challenge while extending the geometrical acceptance to smaller angles. Unfortunately, the performance of state-of-the-art LGAD sensors degrades too quickly with the radiation dose to employ them in high radiation environments where fluences well above $10^{15} 1 \mathrm{MeV} n_{e q} \mathrm{~cm}^{-2}$ are expected within the lifetime of the detector $[9,10]$.

Recently, a new generation of 3D silicon pixel detectors, with distances between the electrodes of few tens of micrometers, have been demonstrated to be resistant to radiation fluences up to $3 \times 10^{16} 1 \mathrm{MeV}_{e q} \mathrm{~cm}^{-2}$ [11]. In these sensors, the electrodes are thin conductive columns engraved in the semiconductor material, which is usually much thicker than the distance between the electrodes. Their time resolution was measured using a ${ }^{90} \mathrm{Sr} \beta$ source and found to be comparable to that of LGAD sensors. Studying its dependence on temperature and applied bias voltage, it was concluded that the resolution is dominated by the effects of inhomogeneities of the electric field, since the large depleted volume traversed by the ionising particles on a path parallel to the electrodes allows one to reduce the Landau fluctuations, and the signal-to-noise ratio is sufficiently large to make the jitter contribution subdominant [12]. Recently, the TimeSPOT Collaboration has developed 3D Silicon devices with trench-shaped electrodes whose resolution was measured to be 20 ps with a beam of minimum-ionising pions at the Paul Scherrer Institut (PSI), in Villigen [4].

Since the spread in the collection time is strictly related to the carrier drift velocity, and therefore to the semiconductor material, an interesting area of research on timing pixel sensors consists in the study of semiconductors other than silicon. In particular, diamond is extensively studied for it combines high carrier velocity with an excellent radiation resistance, which is further improved when a $3 \mathrm{D}$ design is adopted to reduce the drift path [13-17]. Differently from the silicon devices, where the electrodes are obtained with micromachining and doping, in diamond sensors the electrodes are usually obtained focusing a femtosecond laser within the diamond bulk to induce a local phase transition to an ohmic mixture of diamond-like carbon, amorphous carbon and graphite [18, 19]. Recently, the single-hit efficiency of 3D sensors constructed with poly-crystalline chemical vapour deposition (CVD) diamond has been measured in a beam test using a high precision tracking telescope and found to be above $99 \%$ for particle fluxes up to $20 \mathrm{MHz} / \mathrm{cm}^{2}$ and fluences up to $8 \cdot 10^{15} 1 \mathrm{MeV} n_{e q} \mathrm{~cm}^{-2}$ [20].

The high intrinsic break-down voltage allows operating diamond sensors with high electric field which, in combination with the high electron and hole mobility, may result into negligible contribution from the charge collection mechanism to the time resolution of $3 \mathrm{D}$ diamond detectors.

With Landau fluctuations limited by the 3D geometry and the spread in the collection time reduced because of the semiconductor material properties, it is reasonable to expect the jitter to dominate the time resolution. The slope of the output signal is directly affected by the time constant of the sensor which can be approximated by the product of the sensor capacitance by the resistance at the input of the amplifier. While the former is significantly smaller than in typical silicon sensors because of the thinner electrodes and smaller dielectric constant, the combination of diamond-like carbon, amorphous carbon and graphite constituting the electrodes makes the resistance at the input of the amplifier several orders of magnitude larger than in silicon detectors [21]. Higher resistance of the electrodes also results into an increase of the Johnson noise and therefore into larger jitter contributions to the time resolution.

In this work, we describe the design, the fabrication and the characterisation of timing 3D diamond sensors, with electrodes obtained through a pulsed laser graphitisation technique improved by the application of adaptive optics to reduce the electrode resistance. Following this introduction, Sections 2 and 3 describe the design and fabrication process, while Section 4 presents the characterisation of the sensor response to laserinduced excitations, $\beta$ rays from ${ }^{90} \mathrm{Sr}$ and minimum-ionising pions. Throughout the paper, a right-handed coordinate system is adopted, with the $z$ axis parallel to the electrodes and the $y$ axis along the vertical.

\section{SIMULATION}

The sensors described in this paper are top-quality single-crystal CVD (sCVD) diamond specimens [22, 23]. The $4.5 \times 4.5 \times 0.5 \mathrm{~mm}^{3}$ samples used in this study were purchased from Element Six ${ }^{1}$.

The pixel pitch is fixed to $55 \mu \mathrm{m} \times 55 \mu \mathrm{m}$ to ease the comparison with silicon sensors compatible with the Timepix readout chip [24], while all other geometrical and physical properties of the sensors were optimised in terms of time resolution by taking advantage of various simulation approaches and experimental tuning campaigns.

${ }^{1}$ https://www.e6.com 

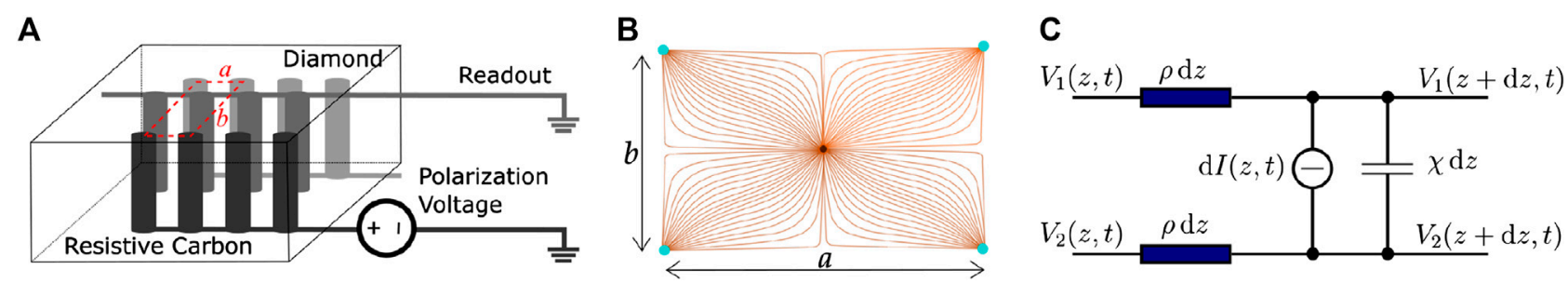

FIGURE 1 | (A) schematic representation of the generic 3D $a \times b$ rectangular elementary cell (not to scale). (B) projection of the cell on the transverse plane, with black and cyan circles representing the electrodes connected by electric field flux lines in brown. (C) electrical model of a single cell used to describe the charge relaxation. The voltage difference between $V_{1}$ and $V_{2}$ encodes the signal generated by an infinitesimal current $\mathrm{d} /$ as propagated through an electrode pair. The electrical parameters of the transmission lines are the distributed resistivity $\rho$ and capacitance $\chi$. Together, the two models for the electric field and the charge relaxation provide a simple description of the 3D sensor.

\subsection{Qualitative Considerations on the Detector Design}

A simplified description of the sensor, modelling the charge collection by the resistive electrodes and the signal relaxation due to the readout electronics, is used to assess the dependence and the relative importance of the design parameters. The resistive electrodes are modeled as infinitely long columns arranged in rectangular elementary cells, as depicted in Figure 1A. The same figure also shows that the readout electrodes, which are usually virtually grounded by the amplifier, are interleaved with polarization electrodes. To simplify the numerical computation, the electric field is assumed to be stationary during the whole process of charge collection. Also the variation of the polarisation bias due to the signal current was neglected. With this approximation, the detector response can be described by a simple two-dimensional model as an application of the Ramo theorem [25]. The 2D representation of the elementary cell used for the simulation is depicted in Figure 1B.

The capacitance of the cell and the resistance of the electrodes are relevant to the propagation of the electrical signal to the input of the readout electronics. A pair of electrodes is modelled as a resistive-capacitive line as illustrated in Figure 1C. The length of the line, and therefore the effect of the line on the signal, depends on the depth at which the charge is generated, providing a third spatial dimension to the description of the sensor.

The resistivity and thickness of the columns, the size of the elementary cell and the inclination of the traversing particles with respect to the sensors are all parameters whose effect can be studied, at least qualitatively, with such a simplified model. In addition, the simulation indicates that larger signals can be obtained connecting the readout and polarization electrodes on the opposite sides of the diamond sample, rather than connecting them to the same side (see Figure 1A).

The simulation also confirms that for higher bias voltages, and therefore higher electric field, the signals are faster and the time resolution improves. The benefit from an increase in the electric field beyond charge carrier velocity saturation is however marginal with respect to lowering the time constant of the charge relaxation process. The order of magnitude of the time constant is fixed by the product of the capacitance of the single cell and the resistance of the electrodes. The capacitance is defined by the size of the elementary cell and by the diameter of the columns. The resistance depends on the quality of the fabrication procedure and, quadratically, on the diameter of the columns.

Small elementary cells are necessary to achieve the spatial granularity required by tracking applications at future large-scale particle detectors. They improve the radiation resistance of sensors as well as their time resolution due to the shorter charge carrier transit time. However, smaller elementary cells result into larger capacitance putting stronger requirements on maximum electrode resistivity.

Finally, the simulation predicts a slight improvement in the time resolution if the trajectory of the ionising particles is inclined by a few degrees with respect to the electrodes.

\subsection{TCAD Simulation of the Sensor}

A more accurate simulation of sensor response has been developed exploiting the numerical simulations within the Technology CAD framework of Synopsys ${ }^{\odot}$ Sentaurus TCAD $[26,27]$. The full simulation relies on the complete $3 \mathrm{D}$ geometry of the elementary cell, on which a complete finiteelement computation of all electrical quantities is performed. To study the effect of geometrical and physical parameters on sensor time resolution, a reliable description of the charge collection mechanism in terms of space and time is of critical importance. Hence, the discretisation of the elementary cell has been carefully designed with a mesh whose granularity increases in the regions where variations of the relevant parameters are larger. The pitch of the simulated sensor is $55 \mu \mathrm{m} \times 55 \mu \mathrm{m}$, and the depth of the diamond sample is $500 \mu \mathrm{m}$. The diameter of the resistive electrodes, modelled as cylinders of conductive diamond-like carbon, is varied between three and $7.5 \mu \mathrm{m}$, corresponding to a single-cell capacitance spanning the range between 26 and $39.4 \mathrm{fF}$. The resistivity of the columns, described within the TCAD environment by an effective reduced band-gap of a diamond-like material, has been varied in a wide range of values between $10^{-7}$ and $10^{21} \Omega \mathrm{cm}$, spanning from metallic to the pure diamond electrodes. The electric field in the bulk depends on the resistivity of the electrodes, because of the voltage drop inside the volume of each electrode. As a consequence, highly resistive electrodes result into electric fields with a non-negligible component along the $z$ direction, especially in the vicinity of the sensor surface. A full 3D model of 

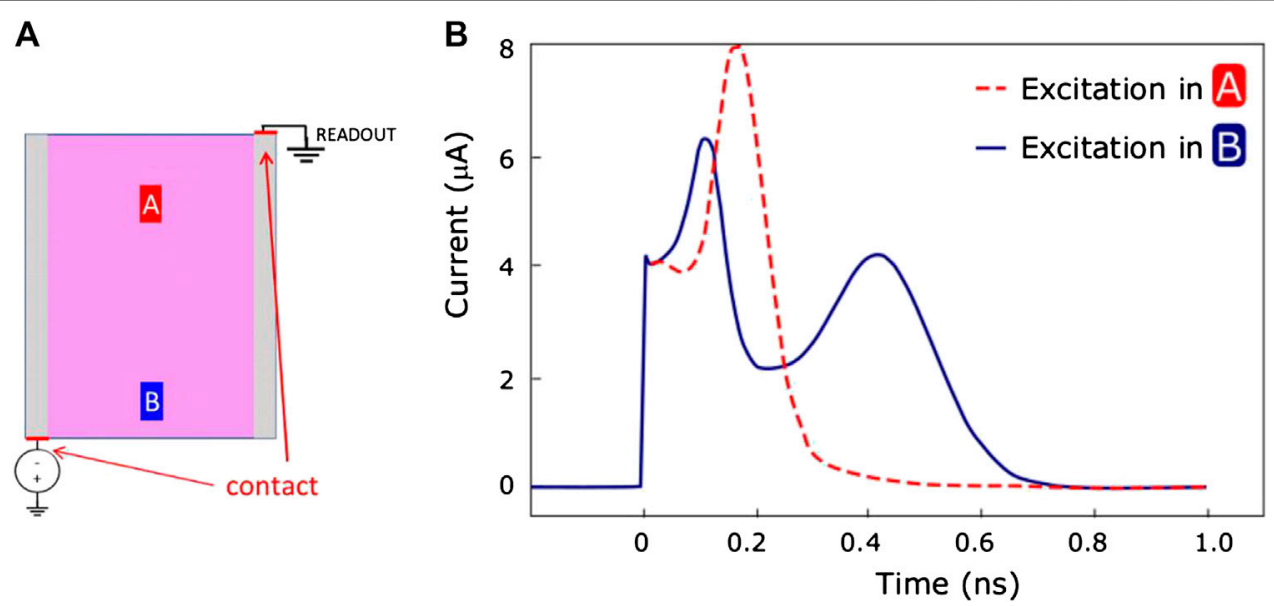

FIGURE 2 | (B) The simulated current response when a charge of $2.88 \mathrm{fC}$ is released in the elementary cell at two different depths, at positions $\mathrm{A}$ (dashed red) and $\mathrm{B}$ (solid blue) as shown in (A)

the electric field within the elementary cell is necessary to take these effects into account.

The complete simulation describes the induced signal together with the signal due to the displacement of the carriers along the resistive electrodes, resulting in a second current pulse with a delay of a few hundreds of picoseconds with respect to the induced signal.

In Figure 2 the shape of the current pulse is shown when a charge of $2.88 \mathrm{fC}$ is released at two different depths of the elementary cell. A common fast leading edge is followed by a depth-dependent structure, faster, as expected, for charge generated closer to the amplifier input. Depending on the applied bias voltage, the carriers collected by the readout electrode can be electrons (for negative polarisation) or holes (for positive polarisation). As the charge relaxation time is dominated by the propagation through the resistive electrodes, the choice of the charge carrier polarity is not expected to affect significantly the time resolution [28]. Therefore, the bias polarisation is chosen by maximising the electric field in either polarities. Junction or tip effects may cause break-down at lower voltage in one of the two configurations, which is then discarded in favour of the other.

\section{FABRICATION}

\subsection{Experimental Setup for Laser Graphitisation}

A Ti:Sapphire femtosecond laser source was used to induce a transformation of the carbon phase from diamond to a mixture of $s p^{2}$ and $s p^{3}$ phases which is sufficiently conductive to behave as a resistive electrode buried in the diamond. The transformation can be induced by thermal excitation or multiple-photon absorption. To engrave electrodes, extremely short laser pulses are preferred to make the non-thermal multi-photon interaction mechanism to dominate and to obtain electrodes thinner and more conductive $[29,30]$.
The sensors here described were obtained with pulse width of $50 \mathrm{fs}$, repetition rate $1 \mathrm{kHz}$, wavelength of $800 \mathrm{~nm}$ and pulse energy of $700 \mathrm{~nJ}$, as measured immediately before impinging in the diamond specimen. A schematic representation of the optical system driving the laser onto the diamond specimen is shown in Figure 3. Two beam splitters were used to arrange a photodiode, a deformable mirror and a CCD camera on the optical path. The laser is focused on the surface or in the bulk of the diamond with a $50 \times$ microscope objective, with numerical aperture of 0.67 , designed for near infrared wavelengths. The same objective projects on the CCD camera a magnified image of the sample during the nanofabrication procedure. A softwarecontrolled deformable mirror was used to correct the beam profile for the spherical aberration introduced by the diamond surface when focusing in the bulk. The voltages applied to a set of forty piezoelectric actuators organised in three concentric sectors define the shape of a reflective film, and thus a space dependent phase shift of the laser wavefront. The image of the mirror is conjugated to the back focal plane of the objective using a set of two lenses with 200 and $100 \mathrm{~mm}$ focal lengths. The piezoelectric actuators are subject to thermal expansion and it was observed that small variation of the room temperature during the day may introduce a curvature on the reflective layer and modify the laser beam divergence. In order to mitigate this effect, a temperature sensor is placed immediately behind the mirror and provides feedback to an automatic temperature controller based on a Peltier cell. The photodiode Thorlabs PDA8GS was used as laser beam monitor and as fast time reference for measurements of sensor response to laser-induced charge generation, as described in Section 4.2. The photodiode signal is acquired by a WaveRunner $610 \mathrm{Zi}$ Lecroy oscilloscope, with $1 \mathrm{GHz}$ bandwidth and maximum sampling rate of $20 \mathrm{Gsps}$.

The sample position was controlled with a three-axis displacement stage driven by computerised stepper motors with a resolution of $350 \mathrm{~nm}$.

A distributed experimental control system software, interfacing the camera, the oscilloscope, the stepper motors, 


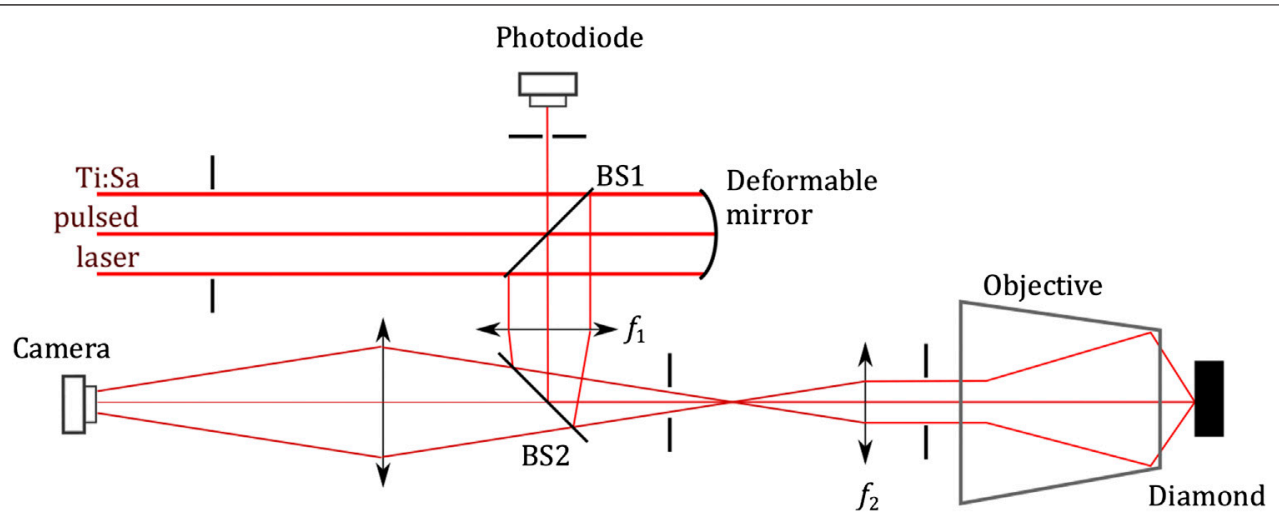

FIGURE 3 | Schematic representation of the optical system. The laser beam is reflected by a deformable mirror which is imaged on the pupil of the objective. The same objective is used to acquire a microscopic image of the sample, during operations, with a CCD camera. A fast photodiode is used to monitor the beam conditions and as time reference for measurements with laser-induced charge generation.

the deformable mirror and its temperature controller was developed and implemented on the Arduino [31] platform. The camera is connected to a computer dedicated to real-time image processing, providing measurements of the laser waist and energy profile with a rate up to $20 \mathrm{~Hz}$. The energy measurement is normalised to the beam intensity as measured by the photodiode.

A client Run Controller application coordinates all the other services, providing a simplified scripting interface to define and run Python routines accessing all the described devices, while hiding the complexity of such a distributed and heterogeneous experimental setup.

\subsection{Spherical Aberration: Description and Correction}

The refractive index mismatch between air and diamond causes spherical aberration in the laser beam focused in the diamond bulk [32]. The effect of the spherical aberration on the focus is

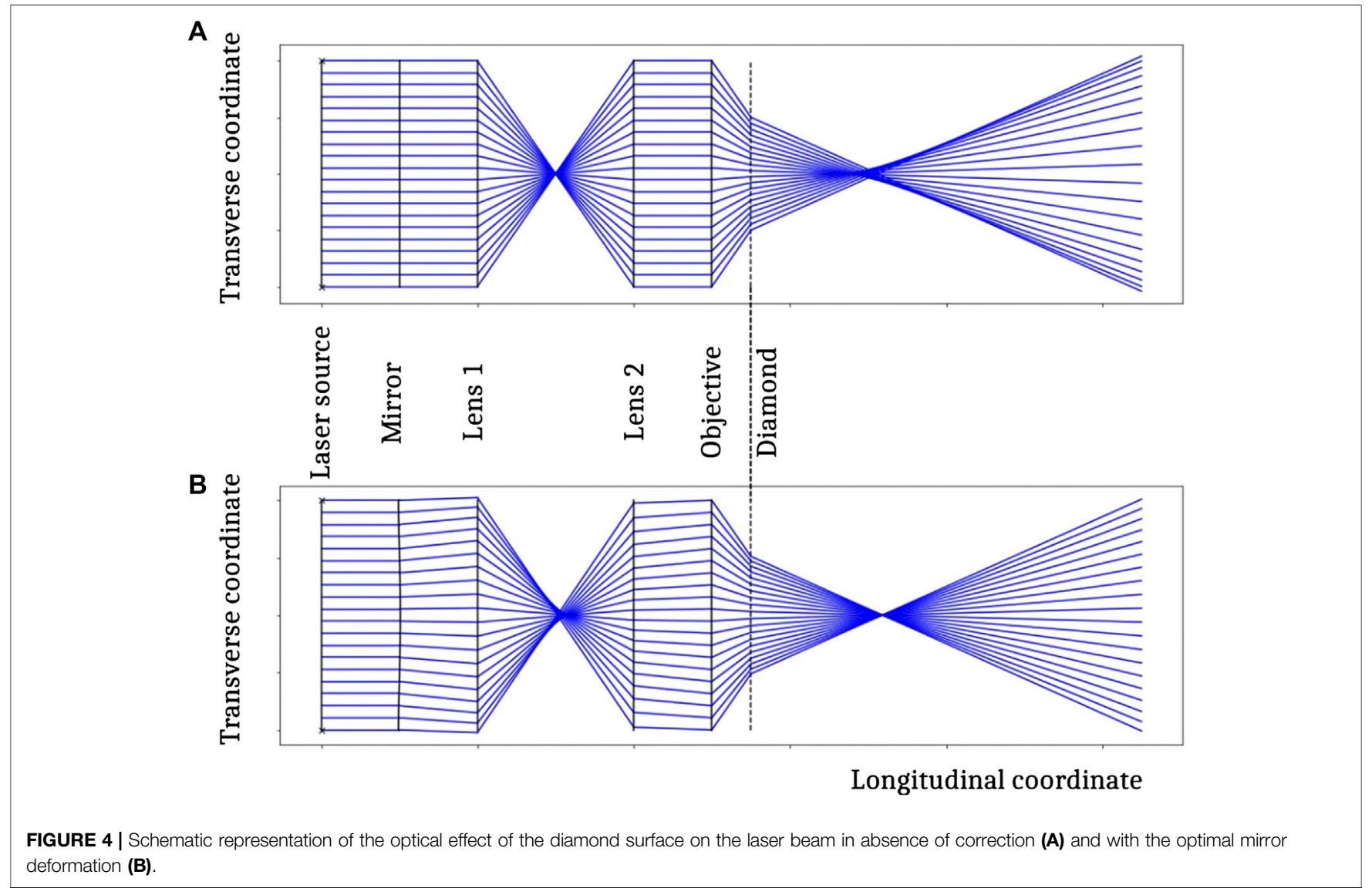


represented in Figure 4. The correction introduced with an adaptive optics element imaged onto the pupil of the objective by a pair of achromatic doublets in a $4 f$ configuration is also shown. Note that to simplify the graphical representation, the effect of the adaptive optics is described in Figure 4 for transmitted rays, whilst the actual correction is defined by a deformable mirror.

In paraxial approximation, the correction of the spherical aberration can be obtained, without modifying the depth in diamond $z$ at which the maximal energy density is concentrated, by applying a phase shift [33].

$$
\psi(\rho ; z)=\alpha z \rho^{2}-\beta z \rho^{4}
$$

to the laser beam, where $\rho$ is the distance to the optical axis and $\alpha$ and $\beta$ are constant parameters to be optimised. In practice, however, we found more convenient to optimise directly the voltages applied to the piezoelectric motors connected to the medium and outer rings of the deformable mirror, indicated as $V_{m}$ and respectively, while the voltage $V_{i}$ applied to the inner sector is fixed to zero.

The optimisation procedure is performed at several depths by maximising the quality of the laser beam spot as diffused by a opalescent coating of a wedge-shaped diamond sample. The optimisation procedure is carried out at different depths in order to infer the optimal values for $V_{m}$ and $V_{o}$ as a function of $z$. The laser source used for this study is the same used to induce the graphitisation, but to avoid damaging the diamond an optical filter is inserted on the optical path to attenuate the intensity by a factor 50 .

The quality of the focus is determined by processing the image $I(x, y)$ as acquired from the camera, corrected for the laser intensity $P$ and the camera exposure time $\Delta t$. The resulting energy distribution is first multiplied by a Gaussian kernel, centered in the most luminous pixel $\left(x_{0}, y_{0}\right)$ and representing the ideal shape of the beam spot, and finally integrated. In formula,

$$
Q=\int \exp \left(-\frac{\left(x-x_{0}\right)^{2}+\left(y-y_{0}\right)^{2}}{2 \sigma^{2}}\right) \frac{I(x, y)}{P \Delta t} \mathrm{~d} x \mathrm{~d} y,
$$

where $\sigma$ is adjusted according to the magnification power of the imaging optical system. To reduce the effect of random fluctuations, the quality of the beam spot is evaluated on several consecutive acquisitions and averaged before using it to define the next mirror configuration to be explored.

The robust Nelder-Mead optimisation method [34], as implemented in the scipy libraries [35], was used to perform the optimisation procedure [36]. The voltages obtained with the optimisation procedure are displayed in Figure 5A as a function of the depth in diamond $z$. Even if the errors are large, especially on the optimal values for $V_{o}$, the dependence of the voltages from the depth is not inconsistent with being linear, and as expected larger corrections are needed when focusing deeper.

A linear fit is performed on the optimised voltages to predict the optimal mirror configuration at a given value of $z$. To validate the complete procedure, we evaluated the relative improvement in the beam spot quality $Q$ obtained with a dedicated numerical optimisation of the mirror shape and with the voltages predicted by the linear model. The results, compared in Figure 5B, are consistent with each other.

The angular coefficients of the linear models predicting the voltages were measured with this procedure several times during the year, after any maintenance intervention on the laser system or devices composing the optical system, and found stable within the uncertainties.

\subsection{Electrode Engraving Procedure and Electrical Tests}

The electrodes are obtained focusing the laser beam on the back of the diamond, i.e., the surface opposite to the beam entrance, and then moving the sample away along the beam direction at a speed of $20 \mathrm{~m} \mu / \mathrm{s}$. The beam focus moves towards the entrance surface of the sample at a speed of $48 \mathrm{~m} \mu / \mathrm{s}$, because of the higher refractive index of diamond, transforming the diamond into resistive carbon phase along the path. While the motor displaces the diamond sample, the deformable mirror is operated to correct for the spherical aberration, modifying its configuration according to the instantaneous position of the beam focus in the diamond.

The resistive columns used as electrodes for the sensor are buried in the diamond by stopping the engraving procedure at $20 \mathrm{~m} \mu$ from the upstream surface. Columns produced to measure the resistivity of the obtained carbon phase are piercing the sample, providing two accessible ends for electrical measurements, surface tracks are engraved at the two ends with a nanosecond laser to ensure good electrical contact.

Figure 6A shows a microscopic image of a set of piercing columns engraved at $100 \mu \mathrm{m}$ from the sample side. The resistance of the columns is measured applying different voltages to the ends of piercing columns and precisely measuring the current. Piercing columns engraved with a threshold-energy laser beam with and without the adaptive optics corrections are compared.

In Figure 6B we report the measurements of the I-V curves for three columns obtained without any correction on the laser beam, and three columns obtained correcting the spherical aberration with the simultaneous displacement of sample and mirror. The improvement is evident, especially in the small-signal region. The $\mathrm{I}-\mathrm{V}$ curves of columns obtained with the corrected beam are found to show a lesser deviation from ohmicity, while the uncorrected beam results into higher dynamic resistance for lower voltages.

\subsection{Sensor Production and Test Electronics}

Two sensors were produced with the system discussed above on sCVD samples. The first, denoted as Sensor A, was produced without any laser beam correction, with a pitch of $100 \mu \mathrm{m} \times$ $160 \mu \mathrm{m}$. The second, named Sensor B, was produced with a pitch of $55 \mu \mathrm{m} \times 55 \mu \mathrm{m}$ to be compatible with the Timepix standard [24] and applying the correction for the spherical aberration.

Sensors A and B were prepared with $9 \times 14$ and $30 \times 30$ elementary cells respectively. Both sensors have an active area of $1.5 \mathrm{~mm} \times 1.5 \mathrm{~mm}$.

The laser beam used to produce Sensor A was adjusted to provide pulses of $1700 \mathrm{~nJ}$ each, while $700 \mathrm{~nJ}$ per pulse were used to produce Sensor B, where finer electrodes were needed to obtain a finer pitch avoiding cracks in the diamond substrate. As a 

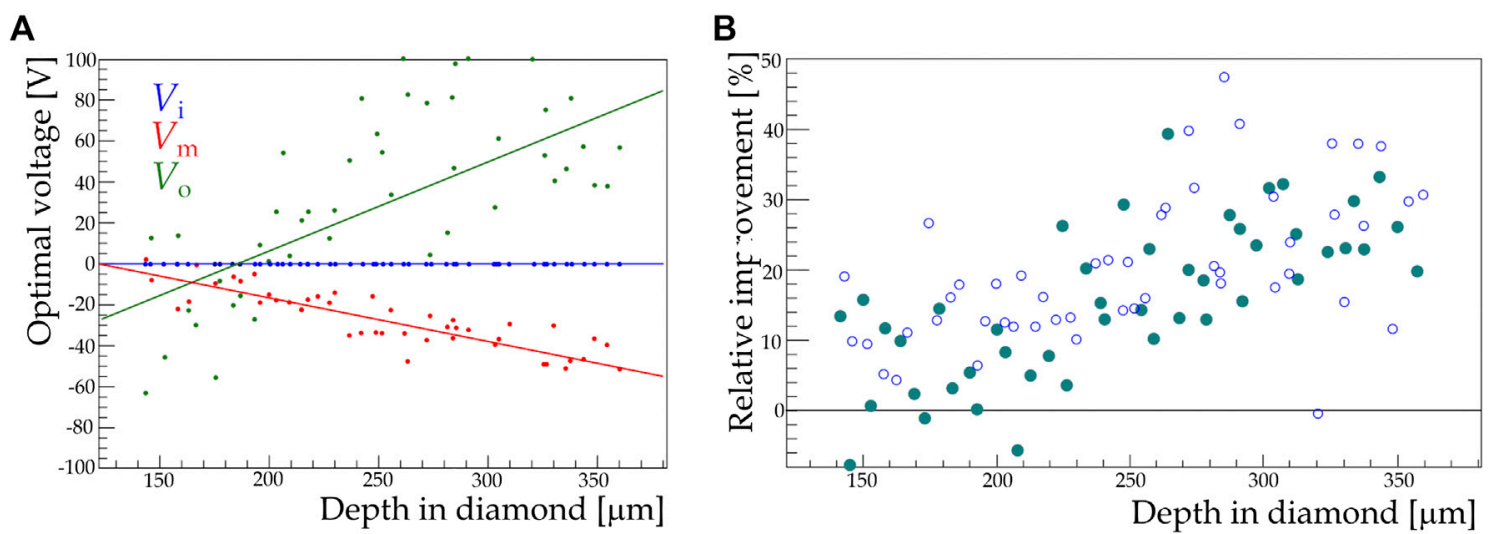

FIGURE 5 | (A) The optimal values for the voltages applied to the piezoelectric motors controlling the deformable mirror as determined with an iterative optimisation procedure. A linear model describing the dependence of the optimal voltage on the depth in diamond is superimposed. (B) the relative improvement in the focus quality $Q$ obtained from direct optimisation (empty markers) or obtaining the voltages as a function of the depth from the linear models shown in (A) (full markers).

consequence, the electrodes of Sensors A and B have diameters of about $30 \mu \mathrm{m}$ and $8 \mu \mathrm{m}$, respectively.

An automated routine was developed to repeatedly engrave the electrodes on a single side. Slow drifts in the laser alignment, typically displacing the focus by few microns in $1 \mathrm{~h}$ of operations, were compensated by manually adjusting the motor position at regular intervals. Engraving $30 \times 30$ cells requires about $8 \mathrm{~h}$, with about $20 \%$ of the time spent for sample alignment, synchronisation and adjustment operations.

To provide test structures of different size and capacitance, surface graphitisation was induced to short circuit sets of different numbers of electrodes. For this operation, a Nd:YAG Q-switched laser source with an $8 \mathrm{~ns}$ pulse width, pulse energies in the range $10-60 \mu \mathrm{J}$, and 1,064 $\mathrm{nm}$ wavelength, was used. A single sensorwide pad was defined for Sensor A, while the electrodes of Sensor B were connected to provide several test structures: a set of strips of $5,9,13,21$, and 30 electrodes, and a larger comb-like pad connecting all the remaining electrodes. Strips are interleaved by elementary cells connected to the comb pad. Surface graphitisation was also used to connect the electrodes to graphitic conductive pads, sputtered with gold to allow wire bonding. Figure 7 reports an optical microscopy image of Sensor A and a schematic representation of the test structures realised on Sensor B.

In the configuration used for the measurements, the sensors are mounted using conductive glue on an electronic board with a hole leaving the sensor active area free in order to minimize the passive material in telescope setups. The gold-sputtered pads on the sensor are connected with $25 \mu \mathrm{m}$ aluminium wires to the input pads of a two stage amplifier with a shaping time of $1 \mathrm{~ns}$ and a bandwidth of $1 \mathrm{GHz}$. A single board, designed at the University of Kansas, hosts eight such readout channels with independent

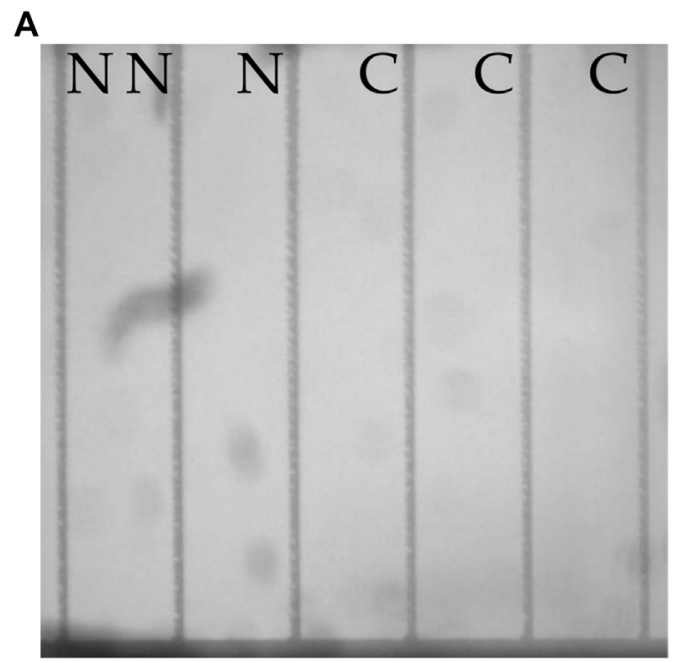

B
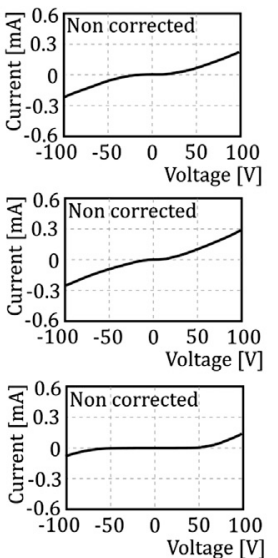
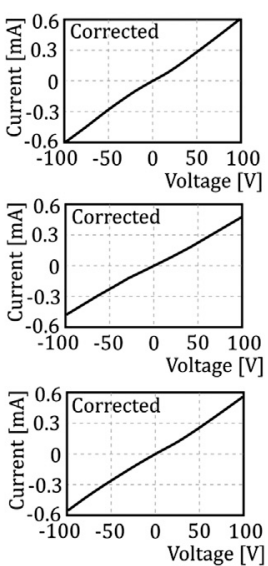

FIGURE 6 | (A) A set of piercing columns with a pitch of $100 \mu \mathrm{m}$ with $(\mathrm{C})$ and without $(\mathrm{N})$ correction, at threshold energy. (B) I-V curves for the piercing columns obtained with and without the optical correction. 
A

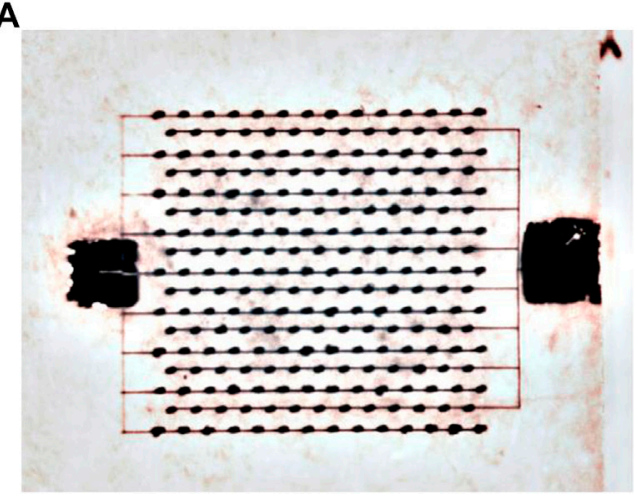

B

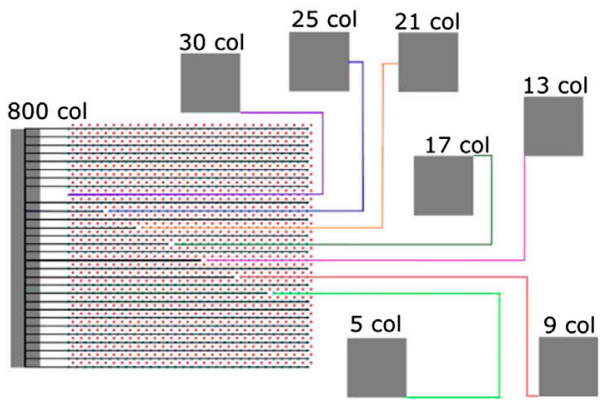

FIGURE 7 | (A) An optical microscopy image of Sensor A.The electrodes are well visible as thin dark points, while the two lateral pads, on opposite diamond surfaces, are designed to allow wire bonding. (B) A schematic representation of the connections of Sensor B into several test structures, each connected to its wirebonding pad. All the electrodes not part of a strip-shaped test structure are short-circuited and connected to the large comb-like pad on the left of the schematic.

amplification and shaping chains $[37,38]$. A metallic cover can be installed on the board to protect the device from electromagnetic interferences, dusts and mechanical shocks that could damage the bonding wires. An aperture in the cover, aligned with the hole on the board below, reduces the material budget upstream the sensor.

\section{RESULTS}

A complete study of the sensors was performed by measuring the properties of their response to two different radiation sources: a femtosecond laser generating point-like charge and diffuse charge generation provided by charged particles at minimum ionisation releasing energy along their whole path through the diamond. A comparison was made between the results obtained with two different charged particle types: the high energy electrons emitted by ${ }^{90} \mathrm{Sr} \beta$ source and $270 \mathrm{MeV} / c$ pions available at the $\pi \mathrm{M} 1$ beam line at PSI.

In each setup, a fast and reliable time reference allowed us to measure the time resolution of sensors along with the signal-to-noise ratio.

The same readout electronics and DAQ system was used for the three measurements, to facilitate the comparison of the results, although differences in the overall experimental setup, like, e.g., cable lengths and environmental noise, may introduce unavoidable discrepancies which are difficult to correct.

\subsection{Data Acquisition and Processing}

Three different DAQ setups, based on LeCroy WaveRunner oscilloscopes, 610Zi, 8104 and $760 \mathrm{Zi}$-a, were used to acquire the amplified signals obtained with laser, electrons and pions. All oscilloscopes were configured to operate with a $1 \mathrm{GHz}$ bandwidth and $20 \mathrm{Gsps}$. The oscilloscopes were connected to the experiment control system via the LXI protocol, defining the overall configuration of the data acquisition, including the trigger strategy and a unique identifier for the data acquisition run.

The acquired data were stored on a removable memory device accessed through a fast USB or PCIe connection, in the
LeCroy-standard ASCII format, which is then processed offline into a compressed binary format immediately accessible to data analysis.

\subsection{Response to Laser Induced Charge Generation}

The same setup described in Section 3.1 and used to induce the graphitisation of the electrodes, was used to drive an attenuated laser beam onto the polarised diamond sensor, generating charge at a well defined position $(x, y, z)$ inside the diamond bulk. The polarisation was positive and the applied voltage was $100 \mathrm{~V}$, while the laser pulse energy was $15 \mathrm{~nJ}$. It is worth mentioning that only a fraction of the pulse energy is transferred to the diamond through multi-photon quantum interaction. The assessment of the exact amount of generated charge is difficult and not relevant to this study. Contrary to the case of minimum-ionising particles, the charge fluctuations from two identical pulses are negligible.

Two fast photodiodes were installed on the optical path of the Ti:Sapphire laser in order to ensure a reference time measurement substantially more precise than the expected diamond time resolution. One of the two photodiodes was positioned within the optical system used to compress the laser pulses to $50 \mathrm{fs}$, while the other is part of the graphitisation optical setup. The former is found to be more stable during long data taking periods and it is therefore chosen as reference. The latter is sensitive to the slow oscillations of the laser beam and it is therefore only used to set an upper limit on the time resolution of the reference.

The jitter of the measured time difference of the two photodiode signals was found to be less than 20 ps which defines an upper limit on the single photodiode time resolution.

For each measurement, the laser beam was focused in the bulk of the diamond sensor in 2500 randomly chosen positions scanning the volume of four elementary cells. The signals obtained from Sensor A and from the strip-shaped pad of 30 elementary cells of Sensor B are acquired for $5 \mathrm{~s}$ for each of the chosen positions. Even if the laser is operated at a pulse frequency of $1 \mathrm{kHz}$, because of the oscilloscope dead time only about 200 

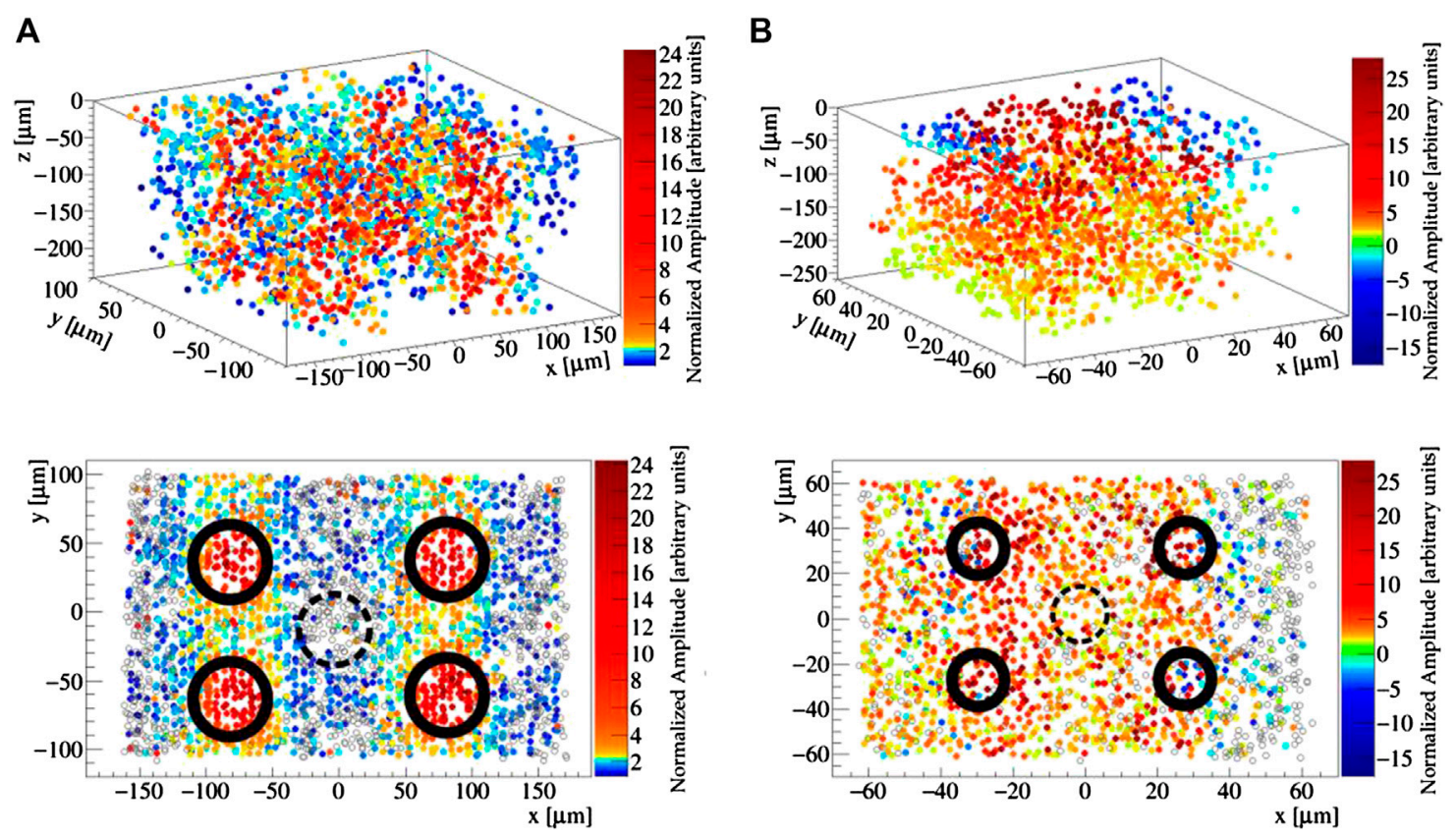

FIGURE 8 | Graphical representation of the dependence of the signal amplitude on the point of excitation of Sensor A (A) and Sensor B (B) with Ti:Sapphire laser pulses. The three-dimensional scans depicted on the top, are projected onto the transverse plane $x y$ in the bottom row. Empty markers indicate measurements where the signal cannot be distinguished from noise. Thick black solid circles indicate the position of the polarisation electrodes, the central dashed circles correspond instead to the readout electrodes.

waveforms are acquired in this interval. Averaging the waveforms acquired for each laser spot position, the noise contribution is reduced and the dependence of the sensor response on the position of the laser spot can be precisely analysed.

Figure 8 reports the amplitude of the averaged signals as a function of the position of the laser excitation for both Sensor A and Sensor B. With a careful choice of the colour code, different regions of the elementary cell are visualised. On Sensor A, we observe large signals for excitations close to the electrodes not connected to the readout electronics. In these regions, the higher electric field causes the signal to be much faster and therefore a larger fraction of the generated charge is integrated by the readout electronics. In the neighbourhood of these electrodes the signal amplitude exceeds the signal amplitude collected in the midpoint between pairs of oppositely polarised electrodes by one order of magnitude. The lack of signal in the uppermost region of the readout electrodes, connected to the electronics, is interpreted as a shadow effect of the superficial graphite connection and of the electrode itself. Thinner columns and smaller elementary cells are beneficial to the uniformity of the response, as shown by the measurement on Sensor B. The position of the electrodes can be barely identified from a mild increase in the amplitude and the signal is observed up to few microns from the electrode. On the other hand, the larger resistance of the electrodes results into an evident dependence of the response on the excitation depth, with the average signal amplitude on the forward surface twice as large as on the backward surface.

The strip-shaped test structure of Sensor B allows one to study effects of cross-talk between neighbouring elementary cells. First of all, we observe a negative signal when exciting the sensor close to the surface, immediately outside of the active area. This effect is well known for silicon detectors and it is due to a capacitive coupling between adjacent elementary cells. This effect is beneficial to the interpretation of Figure 8 because it clearly identifies the borders of the active area of the read elementary cell, as the sharp transition from large positive to large negative signals. Unexpectedly, however, while the position at which we observe negative response follows the symmetry of the elementary cell, we observe different response of the test structure to excitations to the left and to the right adjacent cells. When generating charge in Sensor B at $x$ larger than the cell boundary ( $x>30 \mu \mathrm{m}$ in the coordinate system of Figure 8), we register extremely attenuated signals, mostly indistinguishable from noise. Instead, when generating charge in Sensor B at $x$ less than the cell boundary $(x<-30 \mu \mathrm{m}$ in Figure 8) we observe signals with amplitude of the same order as the signals registered within the elementary cell.

The observed asymmetric cross-talk effect is not fully understood and may depend on local defects in the diamond sample. We notice that, as a consequence of the cross-talk, the description of the averaged response of the test structures to impinging ionising particles discussed in the next sections is subject to an unavoidable background from cross-talk.

Each waveform acquired for each position is processed by a digital Constant Fraction Discriminator (CFD) algorithm to obtain the delay of the signal with respect to the fast trigger signal received from the photodiode. To enhance robustness against the electronic noise, the CFD algorithm scans the 

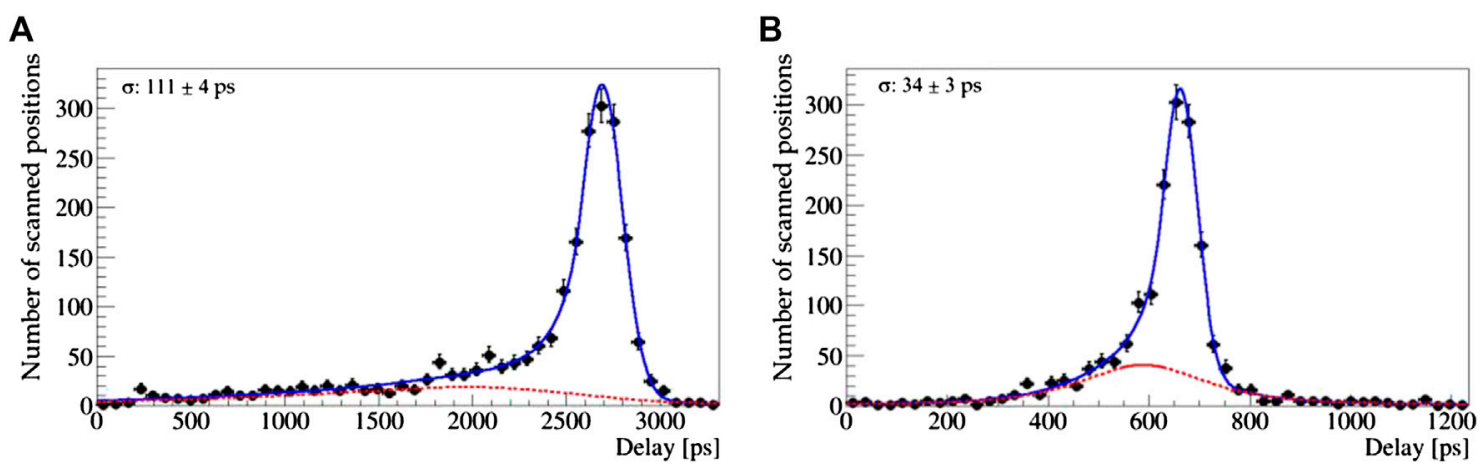

FIGURE 9 | Distribution of the delay between the time reference defined by the photodiode and the time marker on Sensor A (A) and Sensor B (B). The superimposed fit, used to determine the resolution of the main peak, describes the dataset as the combination of a signal component modelled with a Crystal Ball function, and a background component modelled with the empirical function defined in Eq. $\mathbf{3}$.

acquired waveforms to compute and subtract the baseline in order to identify the global maximum of the two samples describing the transition of the waveform through a threshold at $35 \%$ of the maximum signal amplitude, and finally refines the computation of the time marker with a linear fit of the leading edge.

An averaged delay is computed out of the set of about 200 measurements obtained for each scanned position. Since the residual contribution of the electronic noise on the average delay is marginal, the measurement is sensitive enough to allow obtaining the distribution of the delays as a function of the position of the laser spot. Figure 9 shows the distributions of the averaged delays as obtained for Sensor A and the strip-shaped test structure of Sensor B.

Both distributions show an evident non-Gaussian tail at left of the main peak, populated by the samples obtained exciting the sensor in the regions with the highest field, close to the electrodes. In order to quantify the spread of the distribution, we model the dataset as the combination of a signal component modelled with a Gaussian with an asymmetric tail, named Crystal Ball function [39] and an empirical parametrisation of a non-peaking contribution defined as

$$
f(t) \propto \frac{\exp \left(p_{3} t\left(1+p_{4} t\right)\right)}{1+\exp \left(-\frac{t-p_{0}}{p_{1}}\right)}+p_{2},
$$

where $p_{0}, p_{1}, p_{2}, p_{3}$ and $p_{4}$ are free parameters and the normalisation is obtained via numerical integration.

As expected, the width of the main peak is smaller for Sensor B than it is for Sensor A, which indicates that the more homogeneous electric field obtained with fine-pitched thin electrodes is important to control the contribution to the sensor resolution related to the charge collection mechanism.

Throughout the paper, we define the resolution as the standard deviation of the Gaussian core of the Crystal Ball modelling the signal peak.

The obtained resolutions are $111 \pm 4 \mathrm{ps}$ for Sensor $\mathrm{A}$ and $34 \pm 3$ ps for Sensor B.

As expected, resolution is smaller for Sensor B than for Sensor $\mathrm{A}$, this indicates that the more homogeneous electric field obtained with fine-pitched thin electrodes is important to control the contribution to the sensor resolution due to the spread of the collection time.

We notice also that, due to the signal dependence on the generation position, the resolution obtained with the laser excitation mechanism tends to overestimate the contribution from inhomogeneities of the sensor response to the overall sensor resolution with respect to the case of ionising particles. Indeed, with the laser the charge is generated in the single position where the beam is focused on, while for the particles the charge is generated along the whole thickness of the sensor, averaging therefore over different depths and, to a lesser extent, distances from the readout electrode.

\subsection{Response to Electrons From a ${ }^{90} \mathrm{Sr} \beta$ Source}

The response of the sensor to minimum-ionising particles has been measured with electrons from a $270 \mathrm{MBq}{ }^{90} \mathrm{Sr} \beta$ source. In the decay chain ${ }^{90} \mathrm{Sr} \rightarrow{ }^{90} \mathrm{Y} \rightarrow{ }^{90} \mathrm{Zr}$ two electrons are emitted with endpoint 0.546 and $2.28 \mathrm{MeV}$ respectively. The second electron has high enough energy to be considered a minimum-ionising particle. We estimate that the fraction of electrons from ${ }^{90} \mathrm{Y}$ decays with sufficient energy to cross the $500 \mu \mathrm{m}$ thick diamond sample is of the order of $10 \%$. In order to select only highly energetic electrons that behave as minimum-ionising particles in the interaction with the diamond sensor, a fast Cherenkov detector is positioned downstream of the sensor. The detector consists of quartz window coupled to a Micro-Channel Plate Photo-Multiplier Tube (MCP-PMT) produced by Photonis, model PP0365G. The MCP-PMT detector is protected from ambient light with a thick and black low-Z plastic cover that can be easily penetrated by minimum-ionising electrons. Given the high refractive index of the quartz only electrons with $\beta>0.7$ can emit Cherenkov light and produce a signal in the radiator window. Acquiring the signals produced by the diamond sensor in coincidence with the signals from the Cherenkov detector allows selecting electrons with $\beta>0.7 \quad(\beta \gamma>1.0)$ after the ionisation energy loss in the diamond. According to a GEANT4 [40] simulation of the setup, the $\beta \gamma$ factor of the selected electrons is in the range $[2.1,5.7]$ with an average value of 3.2. They can therefore be considered as minimum-ionising particles. The time resolution of the MCP-PMT detector $\sigma_{\mathrm{MCP}}$ was measured in 

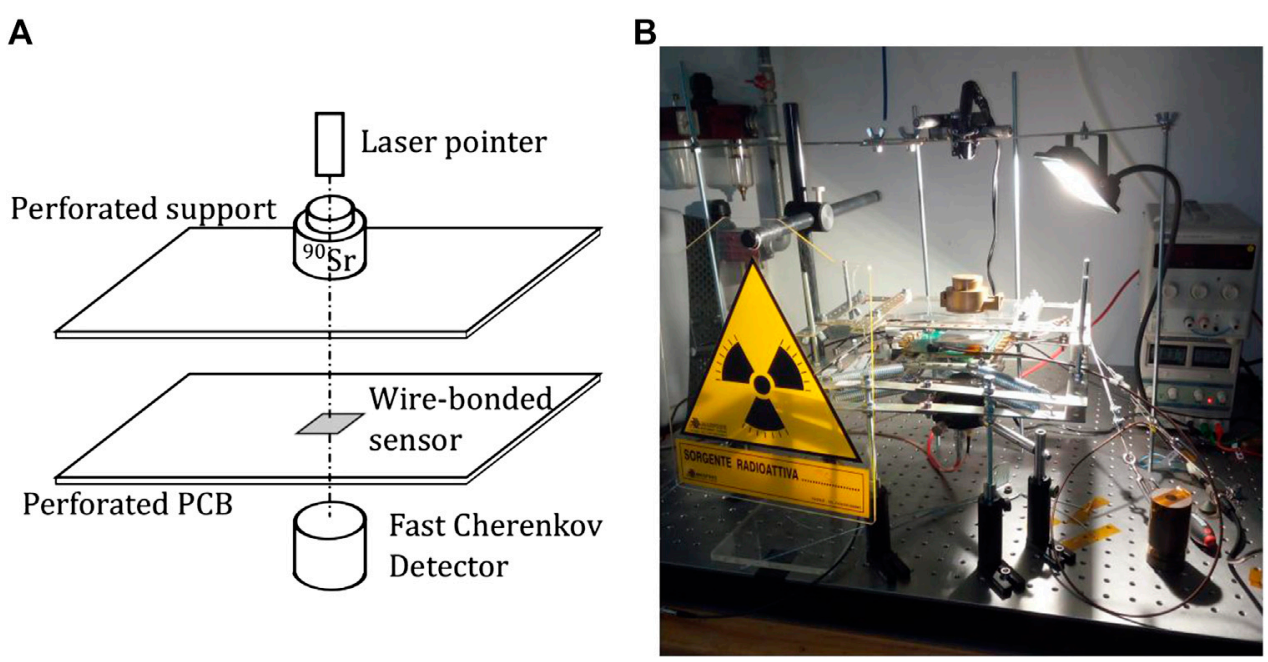

FIGURE 10 | Schematic drawing (A) and picture (B) of the experimental setup used to study the sensor response to electrons from ${ }^{90}$ Sr.

single-photon conditions using laser pulses to be $43 \pm 2 \mathrm{ps}$, consistently with the typical transit time spread declared in the device specifications by Photonis [41]. The experimental setup is depicted in Figure 10.

The acquisition of the sensor signal waveform is triggered by the coincidence of a pulse from the MCP-PMT and a signal from the sensor in a time window of $10 \mathrm{~ns}$. This requirement is necessary to select electrons traversing the sensor, because of the much larger active area of the Cherenkov detector with respect to the test structures in the diamond, which is illuminated by the non collimated ${ }^{90} \mathrm{Sr}$ source.

For each test structure, the sensor trigger threshold is optimised by reducing the noise counting rate to a few percent of the maximum rate allowed by the oscilloscope dead time.

The test structures probed with the $\beta$ source are Sensor A, the strip-shaped pads of Sensor B composed of 5, 9, 21, and 30 elementary cells and the large comb-like pad obtained shortcircuiting the readout electrodes of 822 elementary cells.

For each test structure we acquire data in 6-8 h long runs, corresponding, on average, to $10^{6}$ waveforms. As expected, the trigger rate is significantly lower for small test structures, but since the oscilloscope dead time limits the trigger rate for the larger structures, the acquisition rate does not increase linearly with the number of cells composing the pad.

The analysis of the acquired data and of data from the $270 \mathrm{MeV} / \mathrm{c}$ pion test beam are discussed jointly in the next Section.

\subsection{Response to Minimum-lonizing Pions in a Beam Test}

While the study of the sensor response with $\beta$ rays allows one to acquire large datasets under a large variety of operational conditions, the electrons produced in the decay are subject to significant multiple scattering that can in principle bias the response of the detector. The low energy of the probe particles requires special care in reducing the inactive material traversed by particles in the experimental setup. A complementary study of the response of the detector was therefore performed with a beam of pions with momentum $p_{\pi}=270 \mathrm{MeV} / c$ provided by the $\pi \mathrm{M} 1$ beam line at the PSI in Villigen.

The experimental setup, shown in Figure 11, consists of two Cherenkov detectors with quartz radiators and MCP readout placed downstream of the test structures. To enhance the effectiveness of the study, several test structures of diamond detectors were studied simultaneously. The mechanical structure was designed to allocate up to three $10 \mathrm{~cm}$ wide PCBs upstream of the Cherenkov detectors. To protect the MCP from ambient light, the experimental setup was enclosed in a black box, with sets of connectors to provide power to the electronics, polarisation voltages to the sensors and to connect the output of the amplifiers to the oscilloscopes used for the data acquisition. To reduce the material upstream the senors under test, the beam enters the black box through a $2 \mathrm{~cm}$ wide circular aperture closed by a black plastic film to shield the ambient light.

To mitigate the risk of radiation damage, the oscilloscopes were placed behind a concrete wall and connected to the readout boards with $10 \mathrm{~m}$ long low-loss cables at the cost of a slight worsening of the time resolution due to the attenuation of the signal fast components. This effect was studied in detail and found to be negligible in the determination of the time resolution of diamond sensors.

As in the case of electrons from ${ }^{90} \mathrm{Sr}$, the Cherenkov radiation threshold is used to select relativistic particles, at minimum ionisation. The $\pi \mathrm{M} 1$ beam line is composed by equal momentum particles, predominantly pions, with smaller components of protons, muons and positrons. In particular the proton component is populated by primary protons that lost their energy in the pion production target [42]. These low energy protons do not behave as minimum-ionising particles and tend to release large energy deposits in the sensors, biasing the measurements. These protons are filtered out by inserting on the beam path a $4 \mathrm{~mm}$ thick plastic layer. The residual protons passing the absorbers are too slow to produce Cherenkov 

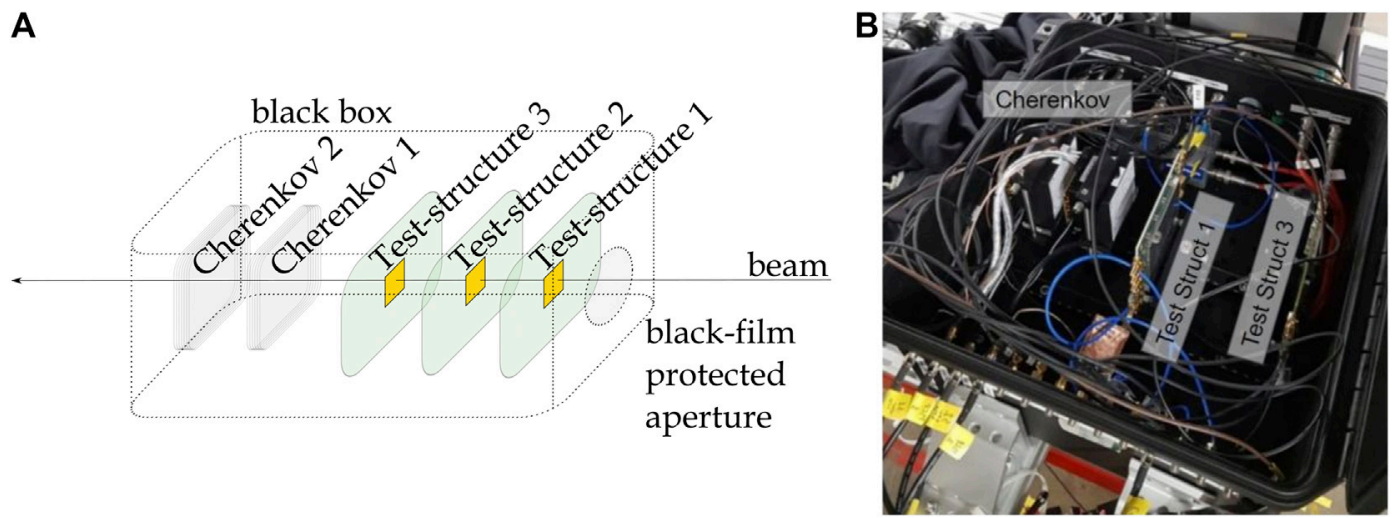

FIGURE 11 | Experimental setup adopted to study the response of the diamond detector to minimum-ionizing pions at PSI. The setup is sketched in (A) and represented in photo in $\mathbf{( B )}$

radiation and are therefore easily rejected by the triggering system. To study the composition of the beam downstream of the absorber, the signal driving the electrical field of the cyclotron accelerator is acquired together with the two MCP-PMT and diamond signals. The time difference $t_{\mathrm{RF}}$ between the cyclotron clock (50 MHz frequency) and the signal measured by the upstream Cherenkov detector (Cherenkov1 in Figure 10) is a measurement of the time of flight of the beam particles, which provides their masses. The distribution of events with respect to $t_{\mathrm{RF}}$, shown in Figure 12, presents four peaks corresponding to four masses of impinging, relativistic particles. Positrons are by far the lightest particles impinging on the Cherenkov detector and therefore can be identified by a larger Cherenkov photon yield, corresponding to a larger amplitude of the signal from the MCPPMT detectors. This allows one to identify the positron peak in the $t_{\mathrm{RF}}$ distribution. The position of the peaks relative to muons, pions and protons was then computed relative to the positron peak. Similar techniques have been employed and validated in the past on the same beam line, using different timing detectors $[38,42]$.
As expected, the dominant contribution is from pions, with a few percent level contamination of muons and positrons, both minimum-ionizing particles, and a sub-permille contribution consistent with the proton mass hypothesis. The latter is interpreted as a background of $\delta$-rays produced in interactions of protons with the black box entrance window. Such lowerenergy electrons are still minimum-ionisation particles, with sufficient velocity to produce Cherenkov light.

To take into account potential small systematic differences among the readout channels connected to the different sensors and electrode layouts that may induce unwanted biases in the results, data have been acquired in two different readout configurations: the first with exactly the same setup as for the test with ${ }^{90} \mathrm{Sr}$ source; the second with readout channels swapped between different test structures.

\subsection{Data Analysis}

The data collected with electrons and pions beams described above are analysed to measure and compare the time resolution of the two sensors.
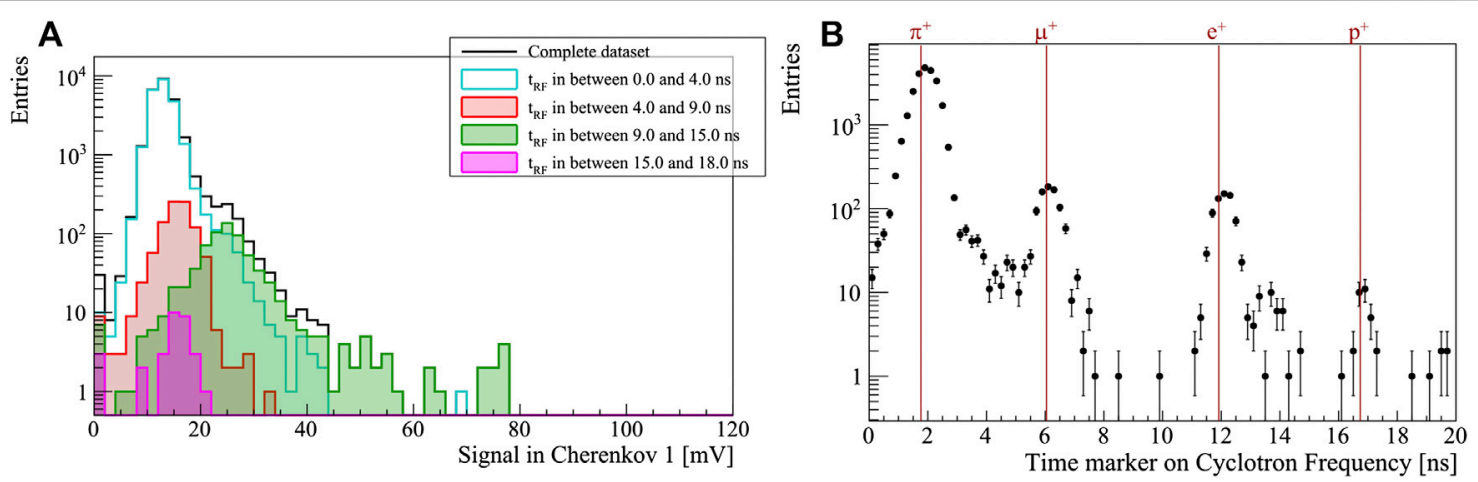

FIGURE 12 | (A) The distribution of the signal from the upstream Cherenkov detectors in four intervals of the relative radio frequency time $t_{\mathrm{RF}}$. (B) The distribution of $t_{\mathrm{RF}}$ in a dataset of $1.5 \times 10^{5}$ waveforms. The four peaks are associated to, from left to right, pions, muons, positrons, and electrons produced in proton interactions downstream the last magnet. The red vertical lines represent the expectations for the four mass hypotheses computed relative to the positrons, whose peak is identified by means of the Cherenkov photon yield. 

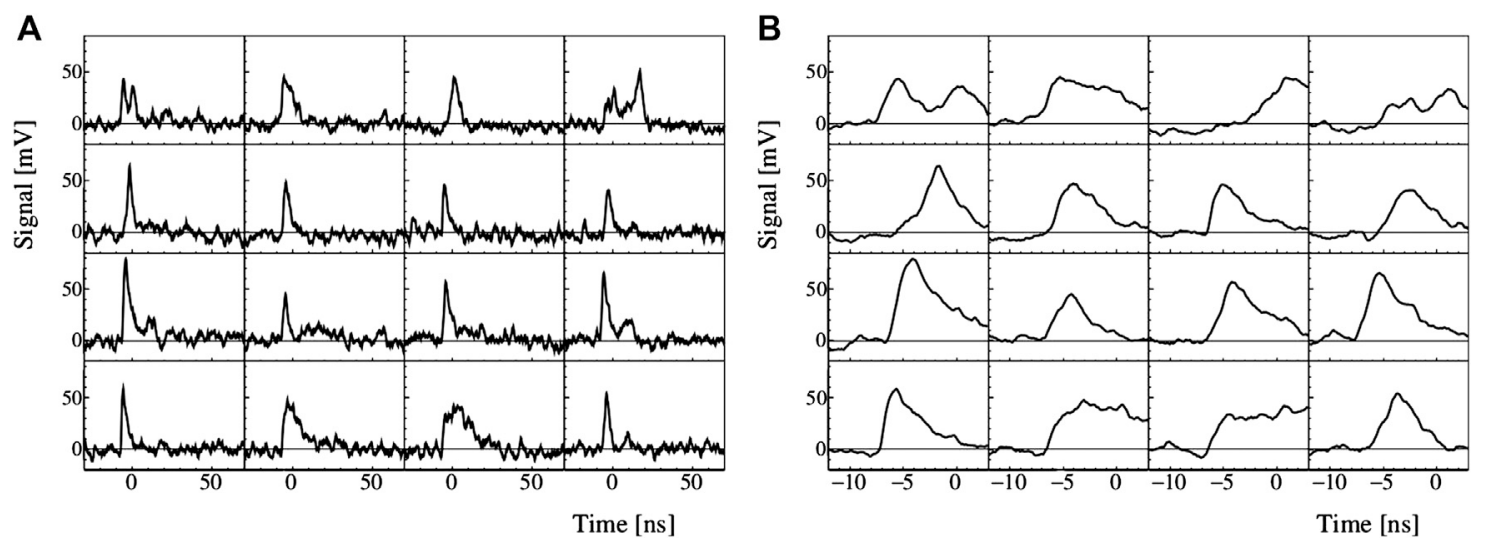

FIGURE 13 | Randomly selected signals obtained from Sensor B strip composed of 30 elementary cells, with an amplitude larger than 40 mV. (A) The full time window is shown, while a zoom on the leading edge of the same events is presented $\mathbf{( B )}$.

The most evident feature of the acquired waveforms is the low signal-to-noise ratio. Figure 13 reports sixteen random examples of waveforms acquired during the beam test with minimumionising pions and the setup described above. Significant signal shape variations are clearly visible notwithstanding the large noise, that introduce undesired time-walk contributions to the timing uncertainty. On the basis of the laser-based studies on the response uniformity within the same elementary cell, such variability can be explained with inhomogeneities of the electric field only partially. Other contributions may be for example the different charge generation mechanism, fabrication differences between electrodes composing the same test structure, and even cross-talk effects.

Two preliminary studies performed on Sensor B prior to any waveform analysis are presented in Figure 14. In the first, the polarisation voltage of the sensor has been varied from -100 to $+125 \mathrm{~V}$ while acquiring at fixed trigger threshold. Outside this voltage range the baseline of the acquired waveforms presents slow and wide oscillations that we interpret as early symptoms of breakdown. A slight asymmetry in the trigger rate between the positive and negative polarisation voltages, favours the former configuration. This might be related to the an asymmetry of the carrier mobility or to defects introducing junction or tip effects. We notice however that other elements of the acquisition chain in addition to the diamond sensor may introduce asymmetries between positive and negative signals. This conclusion is therefore limited to the particular combination of sensors, electronics and oscilloscope used in the analysis. The maximum applicable voltage seems sufficient to reach saturation for negative voltages while a higher voltage may result in higher trigger rate for positive voltages. In both cases, the saturation voltage is close to the breakdown voltage. Considering the large electric field in proximity of the tip at the end of the electrodes, it is reasonable to conclude that the geometrical regions where the charge generation and the breakdown take place are different. The distance between the conductive electrodes and the surface of the diamond limits the polarisation voltage to values that are at most barely sufficient to reach the saturation velocity of the carriers in the whole volume.

A second preliminary study concerns the electronic noise. Because of their large band-gap, the dark current and therefore the shot contribution to the electronic noise is negligible in
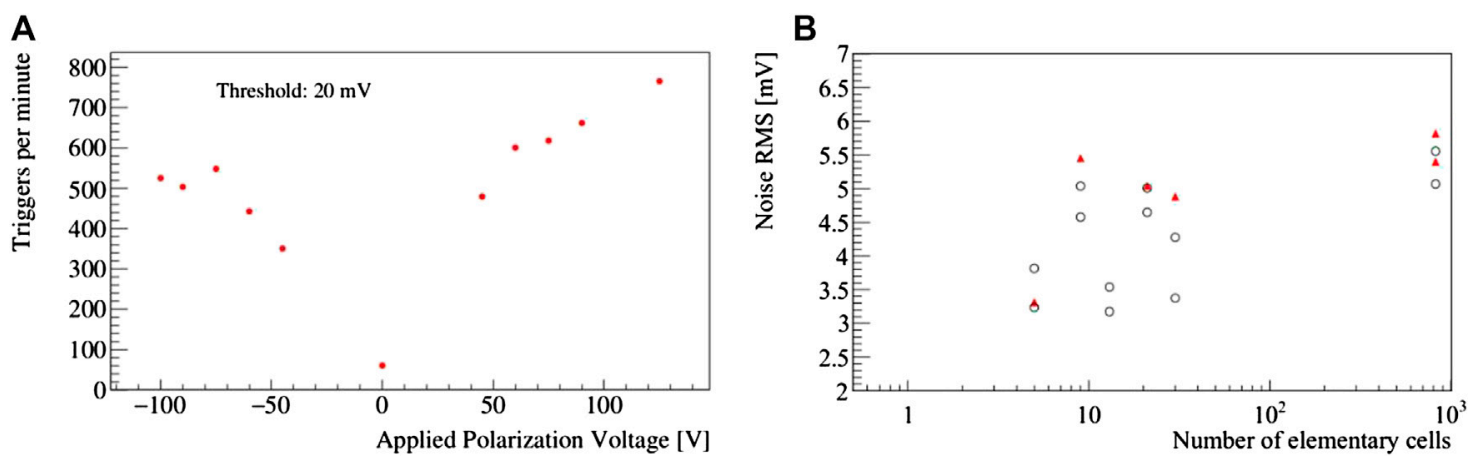

FIGURE 14|(A) The study of the trigger rate as a function of the polarisation voltage. (B) RMS noise as a function of the number of short-circuited elementary cells in several different measurements. In particular, measurements performed during the beam test are represented as red markers, while measurements in the setup with the ${ }^{90} \mathrm{Sr}$ source represented as black empty markers. 


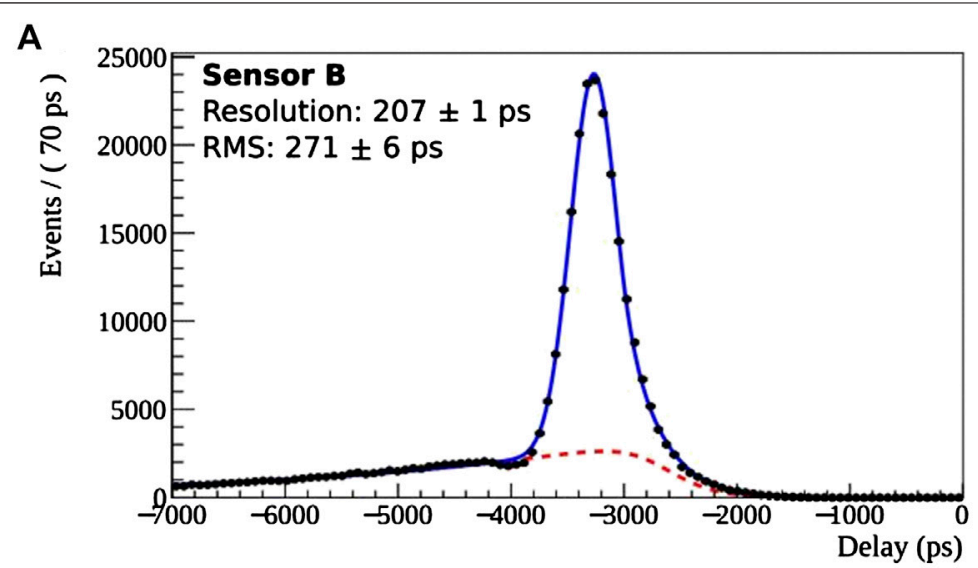

B
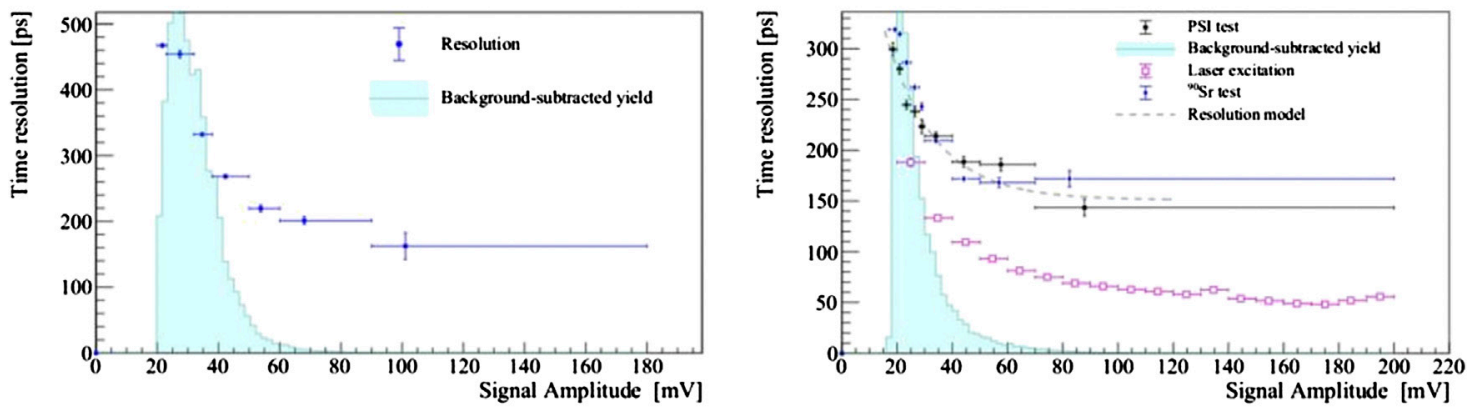

C

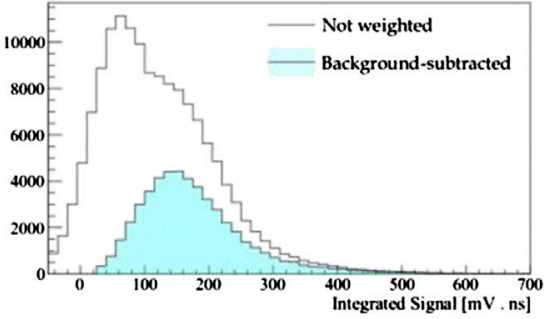

FIGURE 15 | (A) Fit of the delay distribution with a Crystal Ball function superimposed to non-peaking background. (B) Study of the resolution of the sensors A and $\mathrm{B}$, respectively, in bins of the signal amplitude. The histogram of the signal amplitude obtained with the ${ }_{\mathrm{s}} \mathcal{P}$ lot technique applied to the ${ }^{90} \mathrm{Sr}$ dataset is superimposed. For Sensor A, only the resolution obtained with ${ }^{90} \mathrm{Sr} \beta$ rays is reported, while for Sensor B the results obtained with ${ }^{90} \mathrm{Sr}$ electrons, high energy pions and laser excitations are compared. (C) Application of the ${ }_{s} \mathcal{P}$ lot technique to the integrated signal. The white histogram represents the distribution of the integrated signal on the whole dataset, while the cyan histogram is obtained by weighting the events to statistically subtract the background contribution. The 30 -cell strip of Sensor B was taken as an example.

diamond sensors. The large resistance of the electrodes results into a large contribution to the Johnson or thermal noise which is supposed to scale as the square root of the number of elementary cells merged in a test structure. Experimentally, however, no significant correlation between the number of short-circuited electrodes and the measured noise is observed. The tiny capacitance of the elementary cells, estimated of the order of $35 \mathrm{fF}$ per cell is so small compared to the input capacitance of the readout electronics that combining together more than 800 elementary cells results into a barely measurable increase of the noise RMS voltage. The noise RMS voltage measured for Sensor A is perfectly consistent with the values reported in Figure 14 obtained for the various structures of Sensor B. The noise spectrum was analysed and found consistent among the various experimental setups, with negligible contribution from pickup. Dedicated pixel electronics with precise timing capabilities is being developed and will allow for a drastical reduction of the input capacitance of the front-end and hence the noise [43].

Because of the significant cross-talk observed when exciting the diamond sensor with a laser beam as discussed in Section $\mathbf{4 . 2}$ some of the weakest signal events may be interpreted as due to particles traversing the device outside of the active region. Unfortunately, without a precise information on the position of the particle entering the detector it is impossible to measure the detection efficiency or to identify the amplitude distribution of the particles entering the active area of the detector. Since the electrons produced in the ${ }^{90} \mathrm{Sr}$ decay chain have insufficient 
energy to release charge in several planes of a tracking telescope, such a measurement is postponed to a future beam test.

The distribution of the delays between the diamond and reference signals for the 30-cell test structure of Sensor B as obtained with $\beta$ rays is shown as an example in Figure 15B. The result of a fit to the distributions of delays is shown superimposed to the data. The data are fitted with the sum of a Crystal Ball function modelling the peak and the empirical function in Eq. 3 to describe the background of random coincidences.

The same fitting procedure has been repeated in bins of signal amplitude to study the dependence of the time resolution on the signal shape. The dependence of the resolution as a function of the amplitude is presented in Figure 15B, for both Sensor A and B as obtained from ${ }^{90} \mathrm{Sr}$ electron data. For sensor B, the same dependencies obtained with laser and minimum-ionising pions are also shown in Figure 15B; only the results from the 30-cells strip are shown, the results from the other test structures being substantially equivalent. In order to add context to the dependence of the resolution on the amplitude, the distribution of the latter, as obtained with the $\beta$ source setup, is represented on the background of the two plots in Figure 15B. Such a distribution is obtained by applying the ${ }_{s} \mathcal{P}$ lot technique [44] to statistically subtract the background contribution from the signal by computing per-event weights. As an example, in Figure 15C the distribution of the areas of the signals is shown before (empty histogram) and after (filled cyan histogram) background subtraction with this technique.

As expected, the beam test data confirm the results obtained with the $\beta$ source, while the timing of laser excitations is determined with significantly better time resolution because of the different distribution of the generated charge within the diamond. The strong dependence of the resolution on the signal amplitude highlights once more the dominant effect of the electronic noise in the determination of the uncertainty on the timing measurement.

From the comparison of the resolutions obtained with the two sensors (see Figure 15B), a significant improvement is evident when moving from Sensor A to Sensor B. The improvement is confirmed also using different time resolution estimators, including the signal peak FWHM, and the variance of the peak fitted with a Crystal Ball function. The time resolution improvement from Sensor A to sensor B varies between 20 and $40 \%$ depending on the estimation used.

Different algorithms to assign the time marker to the acquired waveforms have been compared. The performance of each algorithm has been evaluated on the basis of the number of signal rising edges correctly identified and on the resulting time resolution. Substantial effort has been spent to find the optimal balance between high detection efficiency and best time resolution. Although details of this study are outside the scope of this paper, a clear outcome is that corrections for the signal leading edge slew rate are needed to significantly improve the time resolution. These corrections seem to confirm the effects of electric field inhomogeneities observed with the laser tests. Its spread seems too large to be due uniquely to noise fluctuations and may result from inhomogeneous electrical field within the sensor, as already pointed out with laser based studies.
To provide another benchmark time resolution estimation for future reference, in addition to the measurement with the digital CFD used throughout this paper, an analog Constant Fraction Discriminator (aCFD) algorithm has been implemented. In the aCFD, a delayed attenuated copy of the signal is subtracted from the signal itself giving as an optimal time marker the zero-crossing time of the resulting bipolar signal. The aCFD provides a complete cancellation of time slewing effects but is sensitive to noise fluctuations that distort the signal, giving rise to false triggers. As a consequence, aCFD offers a better time resolution than the baseline CFD but it shows a decreased tagging efficiency. Indeed using the aCFD (simple CFD) algorithm we obtained a time resolution of $207 \pm$ $1 \mathrm{ps}(263 \pm 1 \mathrm{ps})$ from a sample of $54 \times 10^{3}\left(59 \times 10^{3}\right)$ background-subtracted events recorded with the 30-cell strip of Sensor B and $321 \pm 1$ ps $(389 \pm 1$ ps) from a sample of $9.5 \times 10^{3}\left(13 \times 10^{3}\right)$ background-subtracted events recorded with Sensor A. All the results were obtained using relativistic $(\beta>0.7)$ electrons from ${ }^{90} \mathrm{Sr}$ as probe and the uncertainties are purely statistical.

\section{CONCLUSION AND FUTURE PROSPECTS}

This paper reports on the first detailed study of the time resolution of $3 \mathrm{D}$ pixel diamond sensors. The investigation was carried out by comparing the response of two different sensors to different excitations: minimum-ionising pions, electrons and laser pulses. The main result emerging from this study is the fact that thinner and finer pitch columnar graphite electrodes improve significantly the time resolution, while, at the same time, matching better the requirements in terms of space resolution. Although the timing performances of the diamond devices presented in this work are not yet competitive with those of $3 \mathrm{D}$ silicon sensors, this study has allowed identifying the key elements to proceed towards a further optimisation of these devices, discussed in this Section.

\subsection{Front-End Electronics}

It is evident that the dominant contribution to the time resolution is the electronic noise at the input of the amplifier. The experimental setup with long bonding wires and discrete electronic components seems to introduce dominant noise contributions to the pixel noise, as made evident by the mild dependence of the noise level on the number of short-circuited elementary cells. The development of microelectronics with highresolution timing capabilities is part of the TimeSPOT initiative and its adoption for diamond sensors is expected to introduce a significant, although not yet quantified, improvement of the signalto-noise ratio and hence of the time resolution [43]. While the focus of this work is on the timing capabilities, the choice of the electronics and of the data processing significantly affects the detection efficiency. From the data analysis in this paper, we expect the detection efficiency to be limited by the short integration time of the amplifier. Future work should include a 
direct measurement of the detection efficiency to make such a trade-off evident.

\subsection{Column Resistivity}

The high resistivity of the graphitised columns increases the time constant of the pixel and slows down the extraction of the signal from the bulk of diamond. The progress obtained with an improved focusing of the laser, using adaptive optics to correct the spherical aberration of the wavefront due to the air-diamond interface is evident. However the setup based on a deformable mirror adapted with an iterative procedure based on the laser spot image leads to instabilities that negatively affects the quality of the electrodes and ultimately the sensor time resolution. In particular, we found that small variations of the room temperature result into significant modifications of the mirror curvature, and therefore of the laser divergence. The nanofabrication optical system is being upgraded to replace the deformable mirror with a Liquid-Crystal-on-Silicon Spatial Light Modulator [45], which allows for a much more precise implementation of the theoretically computed phase-shifts necessary to the correct for spherical aberration [33].

\subsection{Column Geometry}

The maximal polarisation voltage was found to be roughly the same on Sensor A and Sensor B, and in general is observed to be roughly independent of the sensor pitch. While unexpected, this result may be understood once the 3D maps obtained with laser excitation are carefully considered. The highest field region is close to the tip of the columnar electrodes: the probability of discharge in this region is clearly independent of the pixel pitch being only affected by the distance between the tip of the electrode and the diamond surface. In other words, the analysed sensors are operated at a voltage which is probably too low to reach the saturation velocity of the carriers within the whole volume, leading to a dependence of signal shape on the position where the charge is generated, worsening the time resolution by effect of an increased spread in the collection time. An optimisation of the geometry close to the end of the electrodes will be carried out and implemented in the next batch of sensors. Improved time resolution obtained in 3D Silicon devices with trench-shaped electrodes [4] suggests to replicate such geometry in diamond sensors, by suitably shaping the nanofabrication laser beam to obtain electrodes with lower resistance and pixels with a

\section{REFERENCES}

1. The ATLAS and CMS Collaborations. Report on the physics at the HL-LHC and perspectives for the HE-LHC. arXiv:1902.10229 (2019)

2. LHCb collaboration. Physics case for an LHCb Upgrade II-opportunities in flavour physics, and beyond, in the HL-LHC era. arXiv.1808.08865 (2018)

3. Sola V, Arcidiacono R, Cartiglia N, Ferrero M, Mandurrino M, Staiano A. Fast timing detectors towards a 4-dimensional tracking. In: PoS (2019) ICHEP2018: 594. doi:10.22323/1.340.0594

4. Anderlini L, Aresti M, Bizzeti A, Boscardin M, Cardini A, Betta GFD, et al. Intrinsic time resolution of $3 \mathrm{D}$-trench silicon pixels for charged particle detection. arXiv:2004.10881 (2020) Accepted by JINST.

5. Pellegrini G, Fernández-Martínez P, Baselga M, Fleta C, Flores D, Greco V, et al. Technology developments and first measurements of low gain avalanche detectors more uniform electrical field. Similarly, a further reduction of the column pitch should also be investigated.

The identification and the solution of the above issues largely defines the next steps of our development of 3D pixel diamond sensors. The results discussed in this paper are already extremely encouraging and represent a further step towards the application of $3 \mathrm{D}$ diamond sensors to tracking detectors with timing capabilities in future experiments at colliders in extreme radiation conditions.

\section{DATA AVAILABILITY STATEMENT}

The raw data supporting the conclusions of this article will be made available by the authors, without undue reservation.

\section{AUTHOR CONTRIBUTIONS}

LA, MB, CC, SL, SM and SS designed and fabricated the sensors; RC, SL, NM, AM, DP and MV developed different parts of the simulations; LA, AC, SL, CL, GP, SS and MV characterised the electrodes and the sensors with table-top measurements; $\mathrm{LA}, \mathrm{AB}$, AC, MG, AdL, AL (10th author), AL (11th author), CL, NM, RM, $\mathrm{SV}$ and MV made the beam test at PSI possible and contributed to the data analysis.

\section{FUNDING}

The research described in this document was funded by INFN as part of the TimeSPOT initiative.

\section{ACKNOWLEDGMENTS}

We acknowledge Patrick Salter for his valuable help with the setup of the aberration-correcting optics, Harris Kagan for enlightening discussion on the front-end electronics, Sara Nocentini for taking care of the surface metallisation of the diamond specimens, Mirko Brianzi and Silvan Stamm for the wire-bonding procedure, and Angela Papa for the support during the activities at PSI.
(LGAD) for high energy physics applications. Nucl Instrum Methods Phys Res Sect A Accel Spectrom Detect Assoc Equip (2014) 765:12-6. doi:10.1016/j.nima.2014.06.008

6. Garcia-Sciveres M, Wermes N. A review of advances in pixel detectors for experiments with high rate and radiation. Rep Prog Phys (2018) 81:066101. doi:10.1088/1361-6633/aab064

7. Cartiglia N, Staiano A, Sola V, Arcidiacono R, Cirio A, Cenna F, et al. Beam test results of a 16 ps timing system based on ultra-fast silicon detectors. Nucl Instrum Methods (2017) A850:83-8. doi:10.1016/j.nima.2017.01.021.

8. Aleksa M, Blomer J, Cure B, Campbell M, D'Ambrosio C, Dannheim D, et al. Strategic R\&D programme on technologies for future experiments (2018) CERN. Technical Report CERN-OPEN-2018-006.

9. Kramberger G, Carulla M, Cavallaro E, Cindro V, Flores D, Galloway Z, et al. Radiation hardness of thin low gain avalanche detectors. Nucl Instrum Methods Phys Res Sect A Accel Spectrom Detect Assoc Equip (2018) 891:68-77. doi:10. 1016/j.nima.2018.02.018 
10. Kramberger G, Baselga M, Cindro V, Fernandez-Martinez P, Flores D, Galloway Z, et al. Radiation effects in low gain avalanche detectors after hadron irradiations. J Inst Met (2015) 10:P07006. doi:10.1088/1748-0221/10/ 07/P07006

11. Lange J, Giannini G, Grinstein S, Manna M, Pellegrini G, Quirion D, et al. Radiation hardness of small-pitch 3D pixel sensors up to a fluence of $3 \times 1016$ $\mathrm{neq} / \mathrm{cm} 23 \times 10^{16} \mathrm{n} / \mathrm{cm}^{2}$. J Inst Met (2018) 13:P09009. doi:10.1088/1748-0221/ 13/09/P09009

12. Kramberger G, Cindro V, Flores D, Hidalgo S, Hiti B, Manna M, et al. Timing performance of small cell 3D silicon detectors. Nucl Instrum Methods Phys Res Sect A Accel Spectrom Detect Assoc Equip (2019) 934:26-32. doi:10.1016/j. nima.2019.04.088

13. Bossini E, Minafra N. Diamond detectors for timing measurements in high energy physics. Front Physiol (2020) 8:248. doi:10.3389/fphy.2020.00248

14. Bauer C, Baumann I, Colledani C, Conway J, Delpierre P, Djama F, et al. Radiation hardness studies of CVD diamond detectors. Nucl Instrum Methods Phys Res Sect A Accel Spectrom Detect Assoc Equip (1995) 367:207-11. doi:10. 1016/0168-9002(95)00545-5

15. Bachmair F, Bäni L, Bergonzo P, Caylar B, Forcolin G, Haughton I, et al. A 3D diamond detector for particle tracking. Nucl Instrum Methods (2015) A786: 97-104 [Erratum: Nucl. Instrum. Meth.A797,331(2015)]. doi:10.1016/j.nima. 2015.03.033.

16. Böni L, Alexopoulos A, Artuso M, Bachmair F, Bartosik M, Beck H, et al. A study of the radiation tolerance of poly-crystalline and single-crystalline CVD diamond to $800 \mathrm{MeV}$ and $24 \mathrm{GeV}$ protons. J Phys D (2019) 52:465103. doi:10. 1088/1361-6463/ab37c6

17. Lagomarsino S, Bellini M, Corsi C, Cindro V, Kanxheri K, Morozzi A, et al. Radiation hardness of three-dimensional polycrystalline diamond detectors. Appl Phys Lett (2015) 106:193509. doi:10.1063/1.4921116

18. Adam W, Bauer C, Berdermann E, Bogani F, Borchi E, Bruzzi M, et al. Development of diamond tracking detectors for high luminosity experiments at the LHC (1996) CERN-LHCC-97-003.

19. Lagomarsino S, Bellini M, Corsi C, Gorelli F, Parrini G, Santoro M, et al. Threedimensional diamond detectors: charge collection efficiency of graphitic electrodes. Appl Phys Lett (2013) 103:233507. doi:10.1063/1.4839555

20. Reichmann M, Alexopoulos A, Artuso M, Bachmair F, Böni L, Bartosik M, et al. New test beam results of $3 \mathrm{~d}$ and pad detectors constructed with polycrystalline cvd diamond. Nucl Instrum Methods Phys Res Sect A Accel Spectrom Detect Assoc Equip (2019) 958:162675. doi:10.1016/j.nima.2019.162675

21. Lagomarsino S, Bellini M, Corsi C, Fanetti S, Gorelli F, Liontos I, et al. Electrical and raman-imaging characterization of laser-made electrodes for $3 \mathrm{~d}$ diamond detectors. Diam Relat Mater (2014) 43:23. doi:10.1016/j.diamond. 2014.01.002

22. Gracio JJ, Fan QH, Madaleno JC. Diamond growth by chemical vapour deposition. J Phys D Appl Phys (2010) 43:374017. doi:10.1088/0022-3727/ $43 / 37 / 374017$

23. Schwander M, Partes K. A review of diamond synthesis by CVD processes. Diam Relat Mater (2011) 20:1287-301. doi:10.1016/j.diamond.2011.08.005

24. Llopart X, Ballabriga R, Campbell M, Tlustos L, Wong W. Erratum to "Timepix, a $65 \mathrm{k}$ programmable pixel readout chip for arrival time, energy and/or photon counting measurements" [Nucl. Instr. and Meth. A. 581 (2007) 485-494]. Nucl Instrum Methods Phys Res Sect A Accel Spectrom Detect Assoc Equip (2008) 585:106-8. [Erratum: Nucl. Instrum. Meth.A585,106(2008)]. doi:10.1016/j.nima.2007.11.003.

25. Shockley W. Currents to conductors induced by a moving point charge. J Appl Phys (1938) 9:635-6. doi:10.1063/1.1710367

26. Morozzi A, Passeri D, Kanxheri K, Servoli L, Lagomarsino S, Sciortino S. Polycrystalline CVD diamond device level modeling for particle detection applications. J Inst Met (2016) 11:C12043. doi:10.1088/1748-0221/11/12/ c12043

27. Morozzi A, Sciortino S, Anderlini L, Servoli L, Kanxheri K, Lagomarsino S, et al. 3D diamond tracking detectors: numerical analysis for timing applications with TCAD tools. J Inst Met (2020) 15:C01048. doi:10.1088/ 1748-0221/15/01/c01048

28. Field JE. The properties of natural and synthetic diamond. In: Crystal Research and Technology. Vol. 28. London, UK: Academic Press (1992) p. 602. doi:10. $1002 /$ crat.2170280504
29. Lagomarsino S, Bellini M, Corsi C, Fanetti S, Gorelli F, Liontos I, et al. Electrical and raman-imaging characterization of laser-made electrodes for $3 \mathrm{~d}$ diamond detectors. Diam Relat Mater (2014) 43:23-8. doi:10.1016/j. diamond.2014.01.002

30. Lagomarsino S, Bellini M, Brianzi M, Carzino R, Cindro V, Corsi C, et al. Polycrystalline diamond detectors with three-dimensional electrodes. Nucl Instrum Methods Phys Res Sect A Accel Spectrom Detect Assoc Equip (2015) 796:42-6. doi:10.1016/j.nima.2015.03.041. Proceedings of the 10th International Conference on Radiation Effects on Semiconductor Materials Detectors and Devices.

31. Mellis D, Banzi M, Cuartielles D, Igoe T. Arduino: an open electronic prototyping platform. Proc. Chi (2007) 2007:1-11.

32. Simmonds RD, Salter PS, Jesacher A, Booth MJ. Three dimensional laser microfabrication in diamond using a dual adaptive optics system. Optic Express (2011) 19:24122-8. doi:10.1364/OE.19.024122

33. Salter PS, Baum M, Alexeev I, Schmidt M, Booth MJ. Exploring the depth range for three-dimensional laser machining with aberration correction. Optic Express (2014) 22:17644-56. doi:10.1364/OE.22.017644

34. Nelder JA, Mead R. A simplex method for function minimization. Comput J (1965) 7:308-13. doi:10.1093/comjnl/7.4.308

35. Virtanen P, Gommers R, Gommers R, Oliphant TE, Haberland M, Reddy T, et al. SciPy 1.0: fundamental algorithms for scientific computing in python. Nat Methods (2020) 17:261-72. doi:10.1038/s41592-019-0686-2

36. Gao F, Han L. Implementing the Nelder-Mead simplex algorithm with adaptive parameters. Comput Optim Appl (2012) 51:259-77. doi:10.1007/ s10589-010-9329-3

37. Minafra N, Al Ghoul H, Arcidiacono R, Cartiglia N, Forthomme L, Mulargia R, et al. Test of ultra fast silicon detectors for picosecond time measurements with a new multipurpose read-out board. Nucl Instrum Methods Phys Res Sect A Accel Spectrom Detect Assoc Equip (2017) 867:88-92. doi:10.1016/j.nima.2017.04.032

38. Minafra N. Development of a timing detector for the TOTEM experiment at the LHC. [PhD thesis]. Bari (Italy): INFN (2016) CERN-THESIS-2016-016.

39. Skwarnicki T. A study of the radiative cascade transitions between the Upsilonprime and Upsilon resonances. [PhD thesis]. Krakow (Poland): Institute of Nuclear Physics (1986) DESY-F31-86-02.

40. Agostinelli S, Allison J, Amako K, Apostolakis J, Araujo H, Arce P, et al. Geant4-a simulation toolkit. Nucl Instrum Methods Phys Res Sect A Accel Spectrom Detect Assoc Equip (2003) 506:250-303. doi:10.1016/S0168-9002(03) 01368-8

41. Garau M. Caratterizzazione di fotorivelatori a micro-channel plate (2016) INFN-THESIS-12094

42. Gilman R, Downie EJ, Ron G, Strauch S, Afanasev A, Akmal A, et al. Technical design report for the paul scherrer institute experiment r-12-01.1: studying the proton radius puzzle with $\mu$ pelastic scattering. arXiv:1709.09753 (2017)

43. Piccolo L. A timing pixel front-end design for hep experiments in $28 \mathrm{~nm} \mathrm{cmos}$ technology. In: 2019 15th Conference on Ph.D research in microelectronics and electronics (PRIME); 2019 July 15-18; Lausanne, Switzerland. IEEE (2019) p. 205-8. Available from: https://ieeexplore.ieee.org/Xplore/home.jsp.

44. Pivk M, Le Diberder FR. sPlot: a statistical tool to unfold data distributions. Nucl Instrum Methods Phys Res Sect A Accel Spectrom Detect Assoc Equip (2005) 555:356-69. doi:10.1016/j.nima.2005.08.106

45. Hamamatsu Photonics KK LCOS-SLM Applications and features. Hamamatsu, Japan: Hamamatsu.com. (2019)

Conflict of Interest: The authors declare that the research was conducted in the absence of any commercial or financial relationships that could be construed as a potential conflict of interest.

Copyright (C) 2020 Anderlini, Bellini, Bizzeti, Cardini, Ciaranfi, Corsi, Garau, Lai, Lagomarsino, Lampis, Loi, Lucarelli, Mariani, Minafra, Morozzi, Mulargia, Passaleva, Passeri, Sciortino, Vecchi and Veltri. This is an open-access article distributed under the terms of the Creative Commons Attribution License (CC $B Y)$. The use, distribution or reproduction in other forums is permitted, provided the original author(s) and the copyright owner(s) are credited and that the original publication in this journal is cited, in accordance with accepted academic practice. No use, distribution or reproduction is permitted which does not comply with these terms. 